\title{
INVARIANT SUBSPACES ON MULTIPLY CONNECTED DOMAINS
}

\author{
Ali AbKar and HÅkan Hedenmalm
}

\begin{abstract}
The lattice of invariant subspaces of several Banach spaces of analytic functions on the unit disk, for example the Bergman spaces and the Dirichlet spaces, have been studied recently. A natural question is to what extent these investigations carry over to analogously defined spaces on an annulus. We consider this question in the context of general Banach spaces of analytic functions on finitely connected domains $\Omega$. The main result reads as follows: Assume that $B$ is a Banach space of analytic functions satisfying some conditions on the domain $\Omega$. Assume further that $M(B)$ is the set of all multipliers of $B$. Let $\Omega_{1}$ be a domain obtained from $\Omega$ by adding some of the bounded connectivity components of $\mathbb{C} \backslash \Omega$. Also, let $B_{1}$ be the closed subspace of $B$ of all functions that extend analytically to $\Omega_{1}$. Then the mapping $I \mapsto \operatorname{clos}(I \cdot M(B))$ gives a one-to-one correspondence between a class of multiplier invariant subspaces $I$ of $B_{1}$, and a class of multiplier invariant subspaces $J$ of $B$. The inverse mapping is given by $J \mapsto J \cap B_{1}$.
\end{abstract}

\section{Introduction}

The study of the lattice of invariant subspaces of a given Banach space $B$ consisting of analytic functions in a domain $\Omega$ of the complex plane $\mathbb{C}$ has attracted much attentions in recent years. This is mainly due to the applications to spectral theory as well as to polynomial approximation theory. Let $\Omega$ be a domain, an open and connected subset of the complex plane, and let $B=B(\Omega)$ be a Banach space of analytic functions defined on $\Omega$. We shall need three different notions of invariant subspaces. A closed subspace $M$ of the Banach space $B$ is said to be invariant provided that $z M \subset M$, where $z$ stands for the

Keywords. Banach spaces of analytic functions, multiplier invariant subspace, index, linear operator, spectrum, holomorphic functional calculus.

1991 Mathematics subject classifications: 47A15, 46E15 (47B38, 30H05). 
coordinate function $z(\lambda)=\lambda$ for every $\lambda$ in $\Omega$ (rather than the usual complex variable). Invariant subspaces are sometimes called simply invariant, or polynomially invariant subspaces. Similarly, we call a closed subspace $M$ of $B$ rationally invariant provided that $r M \subset M$ for every rational function $r$ with poles outside $\bar{\Omega}$ (the closure will always be taken in the extended complex plane $\mathbb{C} \cup\{\infty\}$, whenever we have an unbounded set). Some authors use the phrase fully invariant for rationally invariant subspaces. An analytic function $\varphi$ defined on $\Omega$ is said to be a multiplier for the Banach space $B$, if $\varphi B \subset B$. The set of all multipliers of $B$ is denoted by $M(B)$. A closed subspace $M$ of $B$ is said to be multiplier invariant or hyperinvariant provided that $\varphi B \subset B$, for every $\varphi \in M(B)$. We shall write $M(B)$-invariant for a multiplier invariant subspace when we need to emphasize that $M$ is a multiplier invariant subspace in $B$ (and not in some other space). In this article, we shall mainly deal with multiplier invariant subspaces. It may happen that some of these concepts of invariance coincide for a given space $B$.

Throughout this paper, for $\Omega$ an open and connected subset of $\mathbb{C} \cup\{\infty\}$ with $\bar{\Omega} \neq \mathbb{C} \cup\{\infty\}$, we shall assume that all Banach spaces $B=B(\Omega)$ under consideration satisfy the following axioms:

(0-1) $B$ is a linear subspace of the space of all analytic functions on $\Omega$.

(0-2) The functional of evaluation at $\lambda$ is continuous for all $\lambda \in \Omega$.

(0-3) If $f \in B$, then so is $r f$, for every rational function $r$ with poles in $(\mathbb{C} \cup\{\infty\}) \backslash \bar{\Omega}$.

(0-4) If $f \in B, \lambda \in \Omega$, and $f(\lambda)=0$, then $\exists g \in B$ such that $(z-\lambda) g=f$.

Such conditions have appeared in the literature before. For example, one can find similar set-ups in L. Brown and A. L. Shields $[9]$, and in S. Richter $[\mathbf{2 7}]$.

Axiom (0-3) implies that $M(B)$ contains rational functions with poles outside $\bar{\Omega}$. In case $\Omega$ is a bounded domain, $z$ is regarded as a rational function with pole at infinity so that the polynomials are included in $M(B)$. From the first three axioms together with the closed graph theorem one can obtain the continuity of the operator of multiplication by $z$, that is, $M_{z}=M_{z}[B]: B \rightarrow B$ defined by $f \mapsto z f$. We denote by $\sigma\left(M_{z}\right)$ the spectrum of the operator $M_{z}$, that is, the set of all complex numbers $\lambda$ for which the operator $\lambda-M_{z}$ is not invertible. Assuming that the Banach space $B$ is nontrivial, we conclude by exploiting the last axiom that $\Omega \subset \sigma\left(M_{z}\right)$. Note that $\sigma\left(M_{z}\right)$ consists of all $\lambda \in \mathbb{C}$ for which $(\lambda-z)^{-1} \notin M(B)$. If $\bar{\Omega}$ contains the point at infinity, then $M_{z}$ is not a bounded operator, but we may still talk of $\sigma\left(M_{z}\right)$ as the set of all complex numbers $\lambda$ for which $(\lambda-z)^{-1} \notin M(B)$. It can be shown to be closed in $\mathbb{C}$. 
More generally, we say that a closed subspace $M$ of $B$ is invariant for an operator $T$, defined on $B$, if $T M \subset M$. It is obvious that polynomially invariant subspaces of $B$ are those which are invariant for the operator $M_{z}$. In contrast, multiplier invariant subspaces of $B$ are those which are invariant under all the bounded linear operator $M_{\varphi}: f \mapsto \varphi f$ from $B$ into $B$, where $\varphi \in M(B)$. An invariant subspace of $B$ is called hyperinvariant for the operator $M_{z}$, if it is invariant for all operators which commute with $M_{z}$. For Banach spaces of analytic functions satisfying the axioms (0-1)-(0-4), it is known that the operator $T$ on $B$ commutes with $M_{z}$ if and only if $T=M_{\varphi}$ for some $\varphi \in M(B)$ (see [27, Proposition 2.4]). It follows that the multiplier invariant subspaces of $B$ are precisely the hyperinvariant subspaces for $M_{z}$.

Examples of spaces. Before advancing any further, let us take a look at some familiar Banach spaces of analytic functions which satisfy the above mentioned axioms.

As a first example we mention the classical Hardy spaces of analytic functions on the unit disk $H^{p}=H^{p}(\mathbb{D}), 1 \leq p \leq+\infty$ (for definition see [12]). Note that for $p=+\infty$, we get $H^{\infty}$, the Banach algebra of bounded analytic functions in the unit disk equipped with the supremum norm. It is easy to see that the Hardy spaces satisfy our axioms. The invariant subspaces of the Hilbert space $H^{2}$ were classified by A. Beurling [8]. Later K. deLeeuw and W. Rudin showed that Beurling's result is true for $p=1$, and finally, T. P. Srinivasan extended Beurling's theorem to $H^{p}$, $1 \leq p \leq+\infty[\mathbf{2 2}$, p. 28]. Beurling's theorem is even valid in the context of the spaces $H^{p}$, for $0<p<1$, which are not Banach spaces (however, they are quasi-Banach spaces $[\mathbf{2 5}]$; we use this term to denote a complete $p$-normed space, for some $p, 0<p \leq 1)$. Beurling's theorem asserts that for $0<p<+\infty$, every invariant subspace $M$ of $H^{p}$, other than $\{0\}$, is of the type $M=u H^{p}$ where $u$ is some inner function. Furthermore, if $p=+\infty$, the statement remains true if the word "closed" is replaced by the expression "weak-star closed" (see [13, p. 132]). It is not difficult to extend Beurling's description to general simply connected domains, but the problem of describing the invariant subspaces on mutiply connected domains is more complicated. However, there is a characterization, similar to Beurling's, of rationally invariant subspaces of the Hardy spaces on multiply connected domains. This was observed by D. Sarason [32] for the annulus, and by M. Hasumi [15] and M. Voichick [34], [35] for more general domains (for a brief proof, see the paper of H. L. Royden $[29]$ ). Some effort has been made to describe the simply invariant (but not rationally invariant) subspaces of the Hardy spaces on multiply connected domains. We refer the reader to the works of H. L. Royden [29], D. Hitt [23], V. Yakubovich [36], and A. Aleman and S. Richter [3]. 
Another example of such a Banach space of analytic functions is the Bergman space $L_{a}^{2}=L_{a}^{2}(\mathbb{D})$, consisting of those analytic functions in the unit disk which are square integrable with respect to Lebesgue area measure. More precisely, $f \in L_{a}^{2}$ means that $f$ is holomorphic on $\mathbb{D}$, and has finite norm (we write, for $z=x+i y, d A(z)=\pi^{-1} d x d y$ ):

$$
\|f\|_{L_{a}^{2}}=\left(\int_{\mathbb{D}}|f(z)|^{2} d A(z)\right)^{1 / 2}<+\infty .
$$

The Bergman space $L_{a}^{2}$ is a Hilbert space with the inner product

$$
\langle f, g\rangle_{L_{a}^{2}}=\int_{\mathbb{D}} f(z) \overline{g(z)} d A(z), \quad f, g \in L_{a}^{2}(\mathbb{D}) .
$$

We define $L_{a}^{p}(\Omega)$, for $0<p<+\infty$, the Bergman space with exponent $p$ of a general domain $\Omega$, to be the space of all holomorphic functions $f$ on $\Omega$ such that

$$
\|f\|_{L_{a}^{p}(\Omega)}=\left(\int_{\Omega}|f(z)|^{p} d A(z)\right)^{1 / p}<+\infty,
$$

where $d A(z)$ denotes the area measure on $\Omega$. More generally, we define the weighted Bergman spaces $L_{a}^{p}(\Omega, w)$ where the weight function $w: \Omega \rightarrow \mathbb{C}$ is strictly positive and continuous on $\Omega$ : for $0<p<+\infty$, the space $L_{a}^{p}(\Omega, w)$ consists of those analytic functions $f$ on $\Omega$ for which

$$
\|f\|_{p, w}=\left(\int_{\Omega}|f(z)|^{p} w(z) d A(z)\right)^{1 / p}<+\infty .
$$

For $1 \leq p<+\infty$, this is a norm which makes $L_{a}^{p}(\Omega, w)$ a Banach space. For $w \equiv 1, \Omega=\mathbb{D}$, and $p=2$, we get the previously encountered Bergman space $L_{a}^{2}(\mathbb{D})$. For $1 \leq p<+\infty$, the weighted Bergman spaces satisfy our axioms (0-1)-(0-4). For instance, the continuity of point evaluation functionals follows from the sub-mean value property for subharmonic functions. As we mentioned earlier, Beurling's theorem describes the invariant subspace structure of the Hardy spaces $H^{p}$. In contrast, the invariant subspace structure of the Bergman spaces has never been completely understood and is known to be extremely complicated. However, Aleman, Richter, and Sundberg have recently obtained a genuine analog of Beurling's theorem for $L_{a}^{2}[\mathbf{5}]$.

As a final example of spaces that satisfy our axioms, we would like to mention the spaces $D_{\alpha}$, for $-\infty<\alpha<+\infty$. Consider an analytic function $f$ in the unit disk, with Taylor series $f(z)=\sum_{n=0}^{\infty} a_{n} z^{n}$. The 
space $D_{\alpha}$ consists of those analytic functions in the unit disk for which the following sum is finite:

$$
\sum_{n=0}^{\infty}(n+1)^{\alpha}\left|a_{n}\right|^{2}<+\infty .
$$

It is a Hilbert space with the inner product

$$
\langle f, g\rangle=\sum_{n=0}^{\infty}(n+1)^{\alpha} a_{n} \bar{b}_{n},
$$

where $a_{n}$ and $b_{n}$ are the coefficients in the Taylor series expansions of $f$ and $g$, respectively. The space $D_{0}$ is the familiar Hardy space $H^{2}, D_{1}$ is the Dirichlet space, and $D_{-1}$ is the Bergman space $L_{a}^{2}$. The Dirichlet space consists of those analytic functions $\mathbb{D} \rightarrow \mathbb{C}$ that map the unit disk onto a Riemann surface of finite area $\int_{\mathbb{D}}\left|f^{\prime}\right|^{2} d A$. Clearly, $f \in D_{1}$ if and only if $f^{\prime} \in L_{a}^{2}(\mathbb{D})$. We refer the reader to $[\mathbf{9}],[\mathbf{3 3}]$, and $[\mathbf{2 7}]$ for a review of known results on the spaces $D_{\alpha}$.

In this paper, we study how the lattice of multiplier invariant subspaces changes with the connectivity of the underlying domain. It follows from our main result that to characterize all multiplier invariant subspaces (of index one) of a Bergman space $L_{a}^{p}(\Omega)$ on a finitely connected smoothly bordered domain $\Omega$, one only needs to solve the problem for the Bergman spaces $L_{a}^{p}\left(\Omega^{\prime}\right)$, where $\Omega^{\prime}$ is a simply connected smoothly bordered domain. We should point out that the study of $L_{a}^{p}\left(\Omega^{\prime}\right)$ is essentially the same as that of $L_{a}^{p}(\mathbb{D})$.

Although we shall mainly be interested in a general geometric situation, we start by describing the particular case of an annulus.

We consider a bounded planar domain $\Omega$, and keep in mind the annulus $A_{\rho}=\{z \in \mathbb{C}: \rho<|z|<1\}$ with $0<\rho<1$ as a typical example of $\Omega$. Now assume that $\Omega_{1}$ and $\Omega_{2}$ are two other planar domains satisfying the following conditions:

(1) $\Omega_{1}$ is bounded,

(2) $\Omega_{2}$ is unbounded and non-dense in the complex plane,

(3) $\Omega_{1} \cup \Omega_{2}=\mathbb{C}$ and $\Omega=\Omega_{1} \cap \Omega_{2}$.

For example, one can think of $\Omega_{1}$ as $\{z \in \mathbb{C}:|z|<1\}$, and of $\Omega_{2}$ as $\{z \in \mathbb{C}:|z|>\rho\}$, where $0<\rho<1$. Suppose that $B$ is a Banach space of analytic functions on $\Omega$ (satisfying the axioms). We assume that $B$ can be written as the direct sum of $B=B_{1} \oplus B_{2}^{0}$, where $B_{1}$ and $B_{2}^{0}$ are 
Banach spaces of analytic functions on $\Omega_{1}$ and $\Omega_{2}$, respectively. More precisely, $B_{1}=B \cap \mathcal{O}\left(\Omega_{1}\right)$ and $B_{2}^{0}=B \cap \mathcal{O}_{0}\left(\Omega_{2}\right)$. Here, $\mathcal{O}_{0}\left(\Omega_{2}\right)$ denotes the subspace of $\mathcal{O}\left(\Omega_{2}\right)$ consisting of those functions $f$ such that $f(z) \rightarrow 0$ as $|z| \rightarrow+\infty$. We shall also assume that the multiplier space $M(B)$ can be written as the direct sum of $M\left(B_{1}\right)$ and $M_{0}\left(B_{2}\right)$, where this latter consists of those elements of $M\left(B_{2}\right)$ having the value 0 at infinity. We wish to study the lattices of multiplier invariant subspaces of $B$ and of $B_{1}$. Our main objective is to compare the lattice structure of multiplier invariant subspaces of $B$ with that of $B_{1}$. Once this is done, we would simultaneously have a knowledge of the lattice structure of multiplier invariant subspaces of $B_{2}$ as well, because we can easily interchange $B_{1}$ and $B_{2}$ via a Möbius transformation. The advantage of this kind of comparison theorem is due to the fact that $B_{1}$ is defined on a domain with fewer holes, and so it is likely that its lattice structure is better known than that of $B$.

Suppose that $J$ is an $M(B)$-invariant subspace; then the contraction $J \cap B_{1}$ is an $M\left(B_{1}\right)$-invariant subspace. Conversely, if $I$ is an $M\left(B_{1}\right)$-invariant subspace, then the extension $\operatorname{clos}(I \cdot M(B))$ is an $M(B)$ invariant subspace. Here "clos" indicates closure, and $I \cdot M(B)$ stands for

$$
I \cdot M(B)=\operatorname{span}\{f \varphi: f \in I, \varphi \in M(B)\},
$$

that is, the set of finite linear combinations of products of the form $f \varphi$, $f \in I$, and $\varphi \in M(B)$. The extension $\cos (I \cdot M(B))$ is the smallest multiplier invariant subspace of $B$ containing $I$. It turns out that for a large class of subspaces $I$ and $J$, multiplier invariant in $B_{1}$ and $B$, respectively, the following equalities hold:

$$
I=\operatorname{clos}(I \cdot M(B)) \cap B_{1} \quad \text { and } \quad J=\operatorname{clos}\left(\left(J \cap B_{1}\right) \cdot M(B)\right) .
$$

These equalities guarantee a one-to-one correspondence between parts of the two lattices of multiplier invariant subspaces of $B$ and $B_{1}$. To make the correspondence work, we need to assume that $\sigma\left(M_{z}\left[B_{1} / I\right]\right) \subset \Omega_{2}$ and $\sigma\left(M_{z}[B / J]\right) \subset \Omega_{2}$. Here, the notation $\sigma\left(M_{z}[B / J]\right)$ is used for the spectrum of the operator $M_{z}[B / J]: B / J \rightarrow B / J$ defined by

$$
f+J \mapsto z f+J,
$$

where $J$ is an invariant subspace of $B$. Similarly, one defines the operator $M_{z}\left[B_{1} / I\right]$ and its spectrum $\sigma\left(M_{z}\left[B_{1} / I\right]\right)$. It turns out that it is possible to describe the structure of all multiplier invariant subspaces of index one (see Definition 2.1) in $B$ in terms of the corresponding lattices of multiplier invariant subspaces of $B_{1}$ and $B_{2}$. 
This paper generalizes the methods introduced in $[\mathbf{1 6}]$ to establish a correspondence of this type between the closed ideals of $B_{1}$ and those of $B$ in the Banach algebra setting. Related comparison theorems regarding the structure of closed ideals in some Banach algebras can be found in [17], [18]. The results of this paper can be generalized to quasi-Banach spaces (see [1] ). In particular, the results apply to the Bergman spaces $L_{a}^{p}(\Omega), 0<p<1$, as well.

\section{Preliminaries}

For an unbounded domain $\Omega$, we mean by $\mathcal{O}_{0}(\Omega)$ the subspace of $\mathcal{O}(\Omega)$ consisting of those functions $f(z)$ which tend to 0 as $|z| \rightarrow \infty$ within $\Omega$. Note that here the notation $\mathcal{O}(\Omega)$ is used to indicate the vector space of all analytic functions on the domain $\Omega$ regardless of whether $\Omega$ is bounded or unbounded.

Given an invariant subspace $J$ of the Banach space $B$ of analytic functions defined on $\Omega$, we consider the operator $M_{z}[B / J]: B / J \rightarrow B / J$ defined by the following relation:

$$
M_{z}[B / J](f+J)=z f+J, \quad f \in B .
$$

As we shall see later on, this operator and especially its spectrum plays a significant role in our future work. Recall that the notation $\sigma\left(M_{z}[B / J]\right)$ stands for the spectrum of this operator, that is, the set of all $\lambda \in \mathbb{C}$ for which the operator $\lambda-M_{z}[B / J]$ acting on $B / J$, is not invertible. We note that the spectrum $\sigma\left(M_{z}[B / J]\right)$ is a compact non-empty subset of $\bar{\Omega}$ (see [19]). Similarly, if $J$ is a multiplier invariant subspace of $B$ and if $\varphi \in M(B)$, then we define the operator $M_{\varphi}[B / J]: B / J \rightarrow B / J$ by

$$
f+J \mapsto \varphi f+J, \quad f \in B .
$$

Our first three axioms together with the closed graph theorem imply that $M_{\varphi}$ is a bounded linear operator on $B / J$. We would like to mention that the multiplier space $M(B)$ can be made into a Banach space by putting $\|\varphi\|_{M(B)}=\left\|M_{\varphi}\right\|_{\mathcal{L}(B)}$. It follows immediately that

$$
\|\varphi \cdot f\|_{B} \leq\|\varphi\|_{M(B)} \cdot\|f\|_{B}, \quad \text { for every } \quad \varphi \in M(B), f \in B .
$$

Because of its very important role in our future disscusions, we would like to recall some holomorphic functional calculus. Let $\mathcal{O}\left(\sigma\left(M_{z}[B / J]\right)\right)$ denote the algebra of all functions analytic in some neighbourhood of the spectrum of the operator $M_{z}[B / J]$. Note that every such function $h$ is defined and analytic in a corresponding neighbourhood $U_{h}$ of the compact 
set $\sigma\left(M_{z}[B / J]\right)$; strictly speaking, $\mathcal{O}\left(\sigma\left(M_{z}[B / J]\right)\right)$ is thus an algebra of germs of analytic functions. Consider $h \in \mathcal{O}\left(\sigma\left(M_{z}[B / J]\right)\right)$ and let $D_{h}$ be a compact subset of $U_{h}$ such that its interior contains the spectrum $\sigma\left(M_{z}[B / J]\right)$. Now let $\gamma_{h}$ be the positively oriented boundary of the compact set $D_{h}$ consisting of a finite number of piecewise smooth curves, each homeomorphic to the unit circle $\mathbb{T}$. For $\lambda \in \mathbb{C} \backslash \sigma\left(M_{z}[B / J]\right)$ define the operator $H_{J}: \mathcal{O}\left(\sigma\left(M_{z}[B / J]\right)\right) \rightarrow \mathcal{L}(B / J)$ by

$$
h \mapsto H_{J}(h)=\frac{1}{2 \pi i} \int_{\gamma_{h}} h(\lambda)\left(\lambda-M_{z}[B / J]\right)^{-1} d \lambda .
$$

Here, we identify the complex number $\lambda$ and the operator $\lambda \cdot i d$, where $i d$ stands for the identity operator on $B / J$. We also note that in fact $H_{J}(h)=h\left(M_{z}[B / J]\right)$, the image of the operator $M_{z}[B / J]$ under the holomorphic function $h$, under what is customarily called the holomorphic functional calculus. The mapping $h \mapsto H_{J}(h)$ from $\mathcal{O}\left(\sigma\left(M_{z}[B / J]\right)\right)$ into $\mathcal{L}(B / J)$, the Banach algebra of all bounded linear operators on $B / J$, is linear and multiplicative (see [26, p. 84] or for an interesting discussion of general spectral theory [11]). That is, for functions $h$ and $g$ analytic in a neighbourhood of the spectrum of the operator $M_{z}[B / J]$, we have $H_{J}(h g)=H_{J}(h) H_{J}(g)$. Note that for $\lambda \notin \sigma\left(M_{z}[B / J]\right)$, the inverse operator

$$
\left(\lambda-M_{z}[B / J]\right)^{-1}: B / J \longrightarrow B / J
$$

exists, and that by the analyticity in the $\lambda$ variable the integral defining $H_{J}(h)$ does not depend on the particular choice of $\gamma_{h}$, so that there is no ambiguity in the above integral formula. Assume that the constant function $1 \in B$, so that $1+J \in B / J$. Associate to each $S \in \mathcal{L}(B / J)$, the element $S(1+J)$ which is in $B / J$. This defines a mapping $P_{J}$ : $\mathcal{L}(B / J) \rightarrow B / J$, which sends

$$
S \mapsto P_{J}(S)=S(1+J) .
$$

We then have a mapping $L_{J}=P_{J} \circ H_{J}$ from $\mathcal{O}\left(\sigma\left(M_{z}[B / J]\right)\right)$ into $B / J$; in fact we have the following chain of mappings

$$
\mathcal{O}\left(\sigma\left(M_{z}[B / J]\right)\right) \rightarrow \mathcal{L}(B / J) \rightarrow B / J .
$$

More precisely, we define the mapping $L_{J}: \mathcal{O}\left(\sigma\left(M_{z}[B / J]\right)\right) \rightarrow B / J$ by

$$
h \mapsto P_{J}\left(H_{J}(h)\right)=\left(H_{J}(h)\right)(1+J) .
$$


Let $\varphi$ be a multiplier for the Banach space $B$ of analytic functions, which contains the constant function 1 and satisfies our axioms. Since for $f \in B$, both $f$ and $\varphi f$ are analytic functions on $\Omega$, it follows that $\varphi$ itself is an analytic function. We would like to mention that if we do not assume that $1 \in B$, then our axiom (0-4) implies that $\varphi$ is an analytic function. It turns out that the mapping from $(M(B), B)$ into $B$, sending the pair $(\varphi, f)$ to $\varphi f$, induces a module structure on $B$. In other words, $B$ is an $M(B)$-module. For this reason, we call a module-homomorphism any bounded linear mapping on $B$ which preserves this module structure of $B$. More precisely, we make the following definition, in which we shall temporarily use the notation $A$ for a Banach space of analytic functions on a domain $\Omega$.

Definition 1.1. Let $A$ be a Banach space of analytic functions on $\Omega$, and let $Y$ be a Banach space. A bounded linear mapping $L: A \rightarrow Y$ is said to be a module-homomorphism provided that there exists a continuous homomorphism $L_{M}: M(A) \rightarrow \mathcal{L}(Y)$ such that

$$
L(\varphi f)=L_{M}(\varphi) L(f),
$$

for every $\varphi \in M(A)$ and every $f \in A$.

Remark 1.2. If $L$ is a module-homomorphism $A \rightarrow Y$, then the kernel ker $L$ is an $M(A)$-invariant subspace of $A$. To see this, let $f \in \operatorname{ker} L$ and $\varphi \in M(A)$; the space of multipliers of $A$. We then have $L(\varphi f)=$ $L_{M}(\varphi) L(f)$. But $L(f)=0$, therefore $L(\varphi f)=L_{M}(\varphi)(0)=0$, which shows that $\varphi f \in \operatorname{ker} L$. Moreover, if the linear mapping $L$ is onto then the associated homomorphism $L_{M}$ is unique. To see this, assume that $L_{M}^{*}$ is another continuous homomorphism satisfying the requirements of above definition. Then, for a fixed $\varphi \in M(A)$ and any $f \in A$, we have

$$
L_{M}(\varphi) L(f)=L_{M}^{*}(\varphi) L(f) .
$$

Since $L(f)$ can be any element of the Banach space $Y$, we get $L_{M}=L_{M}^{*}$. When we need to emphasize the associated homomorphism $L_{M}$, we shall speak of the pair $\left(L, L_{M}\right)$ as a module-homomorphism pair.

In the special case that the target Banach space is the complex plane, the associated homomorphism $L_{M}$ sends multipliers to the complex numbers, because $\mathcal{L}(\mathbb{C})=\mathbb{C}$.

Definition 1.3. Suppose that $A$ is a Banach space of analytic functions on $\Omega$ containing 1 and that $M(A)$ is the set of all multipliers of $A$. We mean by a module-functional on $A$, a linear functional $\phi: A \rightarrow \mathbb{C}$ with $\phi(1)=1$ such that $\phi$ is a module-homomorphism $A \rightarrow \mathbb{C}: \phi(\varphi f)=$ $\phi_{M}(\varphi) \phi(f)$, for every $\varphi \in M(A)$ and every $f \in A$. 
We now return to our previous notation $B$ for the Banach space of analytic functions on $\Omega$.

Proposition 1.4. Assume that $B$ is a Banach space of analytic functions on $\Omega$ satisying the axioms (0-1)-(0-4), and that $z$ denotes the coordinate function. If $1 \in B$, then for each $\lambda$, the point evaluation is the only module-functional $\phi$ which satisfies $\phi(z)=\lambda$.

Proof: Having fixed $\lambda \in \Omega$, we want to show that $\phi(f)=f(\lambda)$ for every $f \in B$. First, we note that

$$
1=\phi(1 \cdot 1)=\phi_{M}(1) \cdot \phi(1)
$$

Therefore, $\phi_{M}(1)=1$. Secondly, $\phi_{M}(z)=\lambda$, because

$$
\lambda=\phi(z)=\phi(z \cdot 1)=\phi_{M}(z) \cdot \phi(1)=\phi_{M}(z) .
$$

Let $f \in B$ be arbitrarily chosen. Using axiom (0-4), we can write

$$
f=f(\lambda)+(z-\lambda) g \text { for some } g \in B
$$

Note that $z-\lambda$ belongs to the multiplier space $M(B)$. Hence

$$
\begin{aligned}
\phi(f) & =\phi(f(\lambda))+\phi_{M}(z-\lambda) \phi(g) \\
& =f(\lambda) \phi(1)+\left(\phi_{M}(z)-\lambda \phi_{M}(1)\right) \phi(g) \\
& =f(\lambda),
\end{aligned}
$$

and the proof is complete.

We shall bring this section to an end by the following proposition on the essential ingredients of this manuscript.

Proposition 1.5. Assume that $B$ is a non-trivial Banach space of analytic functions satisfying the axioms (0-1)-(0-4) on the bounded domain $\Omega$. Then the following hold:

(a) $\sigma\left(M_{z}\right)=\bar{\Omega}$.

(b) $\mathcal{O}(\bar{\Omega}) \subset M(B) \subset H^{\infty}(\Omega)$, and if $1 \in B$, then we have

$$
\mathcal{O}(\bar{\Omega}) \subset M(B) \subset H^{\infty}(\Omega) \cap B \subset B \subset \mathcal{O}(\Omega)
$$


Proof: To prove part (a), we fix $\lambda \in \Omega$. Since $B$ is non-trivial, there exists a function $f \in B$ which is not identically zero. We may assume that $f(\lambda) \neq 0$. Suppose that $\lambda \in \mathbb{C} \backslash \sigma\left(M_{z}\right)$, then $\lambda-M_{z}$ as an operator on $B$ is invertible and in particular, it is onto. That is, there exists a function $g \in B$ such that $(\lambda-z) g=f$. But this is not possible, because $f(\lambda) \neq 0$. Thus $\Omega \subset \sigma\left(M_{z}\right)$, and since the spectrum of $M_{z}$ is compact, we get $\bar{\Omega} \subset \sigma\left(M_{z}\right)$. For the reverse inclusion, suppose that $\lambda \notin \bar{\Omega}$; then by axiom (0-3), we have $1 /(\lambda-z) \in M(B)$. Now it is easily seen that the operator $\lambda-M_{z}$ is invertible, so $\lambda \notin \sigma\left(M_{z}\right)$ which proves the desired inclusion. Thus part (a) is proved.

Proof of $(b)$ : For the first inclusion we shall use the holomorphic functional calculus. Let $\varphi \in \mathcal{O}\left(\sigma\left(M_{z}\right)\right)$, and let $f \in B$. We intend to show that $\varphi f \in B$. Using the holomorphic functional calculus, we get $\varphi\left(M_{z}\right) \in \mathcal{L}(B)$. Note that here, we use the assumption that $\varphi$ is holomorphic in a neighbourhood of the spectrum of the operator $M_{z}$. Choosing an arbitrary function $f \in B$, we have $\varphi\left(M_{z}\right) f \in B$. What therefore remains to be proved is that $\varphi\left(M_{z}\right) f=\varphi f$. To this end, we first recall that

$$
\varphi\left(M_{z}\right)=\frac{1}{2 \pi i} \int_{\partial U} \varphi(\lambda)\left(\lambda-M_{z}\right)^{-1} d \lambda
$$

where $U$ is a finitely connected smoothly bordered domain containing $\sigma\left(M_{z}\right)$ and such that $\bar{U}$ lies in the domain of analyticity of $\varphi$, and $\partial U$ is oriented in the positive direction. Hence

$$
\begin{aligned}
\varphi\left(M_{z}\right) f & =\frac{1}{2 \pi i} \int_{\partial U} \varphi(\lambda)\left(\lambda-M_{z}\right)^{-1} f d \lambda \\
& =\frac{1}{2 \pi i} \int_{\partial U} \varphi(\lambda)(\lambda-z)^{-1} f d \lambda \\
& =\varphi f .
\end{aligned}
$$

So far the first inclusion is proved. The facts that $M(B) \subset H^{\infty}(\Omega)$ and that if $1 \in B$, then $M(B) \subset H^{\infty}(\Omega) \cap B$, are the contents of Proposition 2.6 of $[\mathbf{2 7}]$.

\section{Basic Concepts}

Let $M$ be an invariant subspace of $B$ and let $\lambda \in \Omega$. It is known that the dimension of the quotient space $M /(z-\lambda) M$ does not depend on $\lambda[\mathbf{2 7}]$. Therefore, we can make the following definition.

Definition 2.1. We say that the invariant subspace $M \neq\{0\}$ of $B$ has index $n$ if $\operatorname{dim}(M /(z-\lambda) M)=n$. In particular, if $M$ is the trivial null subspace, then the index of $M$ is 0 . 
The invariant subspaces of index $n$ are also known as invariant subspaces having the codimension $n$ property. Before advancing any further, we turn to a class of examples of invariant subspaces of index one. As we mentioned earlier, we shall assume that the multiplier invariant subspace $J$ satisfies the condition $\sigma\left(M_{z}[B / J]\right) \subset \Omega_{2}$. This entails that $J$ has index one (see Corollary 2.4). Hence we need to have a reasonable class of examples.

Examples of index one invariant subspaces. For a subset $S$ of $B$, the notation $[S]$ is used for the smallest invariant subspace of $B$ which contains $S$. If $S$ consists of just one single element $f$, then the notation $[f]$ is used for the smallest invariant subspace of $B$ containing $f$. Such invariant subspaces are called cyclic or singly generated. Note that $[f]$ is the closure of the polynomial multiples of $f$ in $B$. Similarly, one may consider $M[f]$, the closure in $B$ of multiplier multiples of $f$. Invariant subspaces of this kind always have index one ([27, Corollary 3.3]). On the other hand, by Beurling's theorem, every invariant subspace of the Hardy space $H^{2}$ is cyclic (see $[\mathbf{1 4}$, p. 82] or $[\mathbf{2 4}$, p. 99]). In fact, unless it is $\{0\}$, it is of the type $u H^{2}=[u]$ for some inner function $u$. Thus every invariant subspace of $H^{2}$ has index one. It is also known that every nontrivial invariant subspace of the Dirichlet space $D_{1}$ has index one. This was noticed by S. Richter and A. L. Shields [28]. This result remains true for weighted Dirichlet spaces, as was observed by A. Aleman [2]. Moreover, by recent work of Aleman, Richter, and Ross [4], many other Banach spaces of analytic functions share this property. However, given $\alpha<0$, and $1 \leq n \leq \infty$, there exists an invariant subspace $M_{n}$ of $D_{\alpha}$ having index $n$, as was observed by Apostol, Bercovici, Foias, and Pearcy [6]. The Bergman space $L_{a}^{2}$ is the special case $\alpha=-1, L_{a}^{2}=D_{-1},[6],[7]$. The proofs presented for the existence of invariant subspaces of a given index in both of the last two examples have abstract natures involving the axiom of choice. A concrete construction of such invariant subspaces was first found by H. Hedenmalm [20] for the Bergman space $L_{a}^{2}$, and then by H. Hedenmalm, S. Richter, and K. Seip [21] for the Bergman spaces $L_{a}^{p}$.

In any Banach space of analytic functions satisfying our axioms, every invariant subspace of index larger than 1 contains an invariant subspace of index 1. Also every closed ideal in a unital Banach algebra, for instance $H^{\infty}$, has index one [27]. The spaces $D_{\alpha}$ are algebras for $\alpha>1$. Since the polynomials are dense in $D_{\alpha}$, every invariant subspace of $D_{\alpha}$ for $\alpha>1$ has index one [27]. This is so even for $0<\alpha<1$ (see [4, p. 260]). Finally, we note that the invariant subspaces of index 1 form a complete lattice with respect to intersection and span $[\mathbf{2 7}]$. 
Definition 2.2. The collection of all $\lambda \in \mathbb{C}$ for which the operator $\lambda-M_{z}[B / J]$ is not onto is denoted by $\sigma_{J}^{\prime}=\sigma^{\prime}\left(M_{z}[B / J]\right)$ and is called the weak spectrum of the operator $M_{z}[B / J]$.

It is clear that the weak spectrum $\sigma_{J}^{\prime}$ is a subset of the spectrum $\sigma_{J} \subset \bar{\Omega}$. For an invariant subspace $J$ we have in general $\sigma_{J}^{\prime} \cap \Omega=Z(J)$, where the notation $Z(J)$ stands for the common zero set of $J$ in $\Omega$, that is, the intersection of zero sets of all the elements of $J$ (see $[\mathbf{2 7}$, Proposition 4.4]). So far we know that $\sigma_{J}^{\prime} \cap \Omega \subset \sigma_{J} \cap \Omega$. Now, assume that the invariant subspace $J$ has index one and that $\lambda \in \Omega \backslash \sigma_{J}^{\prime}$, so that the operator $\lambda-M_{z}[B / J]$ is onto. We claim that it is also oneto-one. To see this, let $\left(\lambda-M_{z}[B / J]\right)(f+J)=0+J$, which implies that $(\lambda-z) f \in J$. Now, we use Lemma 3.1 of $[\mathbf{2 7}]$ to obtain $f \in J$, or equivalently $f+J=0+J$ which in turn proves that $\lambda-M_{z}[B / J]$ is one-to-one. Therefore, $\lambda \in \Omega \backslash \sigma_{J}$, making $\sigma_{J}^{\prime} \cap \Omega=\sigma_{J} \cap \Omega$ (see [27, Theorem 4.5]). An alternative way to define the weak spectrum is the following: $\lambda \in \mathbb{C} \backslash \sigma_{J}^{\prime}$ if and only if $(\lambda-z) B+J=B$. The following proposition sheds some light on the relationship between the spectrum of the operator $M_{z}[B / J]$ and the index of $J$.

Proposition 2.3. Assume that $B$ is a Banach space of analytic functions satisfying the axioms (0-1)-(0-4) on $\Omega$. If $J$ is an invariant subspace of $B$ such that index $(J)>1$, then we have $\sigma\left(M_{z}[B / J]\right)=\bar{\Omega}$.

Proof: We know that for $\lambda \in \Omega, \operatorname{dim}(J /(\lambda-z) J)=\operatorname{index}(J)$ and also

$$
\sigma\left(M_{z}[B / J]\right) \subset \sigma\left(M_{z}\right)=\bar{\Omega} .
$$

For the reverse inclusion, it suffices to show that $\Omega \backslash Z(J) \subset \sigma\left(M_{z}[B / J]\right)$. Assume that $\lambda-M_{z}[B / J]$ is invertible, for $\lambda \in \Omega \backslash Z(J)$. Then it is oneto-one, so $\left(\lambda-M_{z}[B / J]\right)(f+J)=0+J$ must imply $f \in J$. But the above equality is equivalent to $(\lambda-z) f \in J$, therefore this latter must imply $f \in J$. Since we have

$$
(\lambda-z) f \in(\lambda-z) B=\{g \in B: g(\lambda)=0\}=M_{\lambda},
$$

we obtain $(\lambda-z) f \in J \cap M_{\lambda}$. But $f \in J$ if and only if $(\lambda-z) f \in(\lambda-z) J$. Now, the question is the same as asking whether we have

$$
(\lambda-z) J=J \cap M_{\lambda} .
$$

This is true for $\lambda \in \Omega \backslash Z(J)$ precisely for invariant subspaces $J$ of index 1 $\left[\mathbf{2 7}\right.$, Lemma 3.1]. This means that $\sigma\left(M_{z}[B / J]\right)$ cannot be smaller than $\bar{\Omega}$. The proof is complete. 
Corollary 2.4. The only possibility for the invariant subspace $J$ of the Banach space $B$ of analytic functions on $\Omega$ to satisfy the condition $\sigma\left(M_{z}[B / J]\right) \neq \bar{\Omega}$ is that $J$ has index one.

Proposition 2.5. Assume that B is a Banach space of analytic functions satisfying the axioms (0-1)-(0-4) on $\Omega$, and that $J$ is a multiplier invariant subspace of $B$. If $\sigma_{J}=\sigma\left(M_{z}[B / J]\right) \subset \Omega$, then there exists a polynomial $p$ with zeros in $\Omega$ such that $J=p B=\{p f: f \in B\}$.

Proof: As we have seen before, $\sigma_{J} \cap \Omega=Z(J)$, so that $\sigma_{J}=Z(J)$. It is evident that $Z(J)$ consists of finitely many points of $\Omega$, otherwise it would accumulate at some point in $\Omega$, in which case the unicity theorem for analytic functions would force $J=\{0\}$, making $B / J=B$, whence $\sigma_{J}=\sigma\left(M_{z}[B]\right)=\bar{\Omega}$, contradicting our assumption. Let $p$ be a polynomial with zeros in $Z(J)$, such that the multiplicity of each zero equals the least common multiplicity of all the functions in $J$. As a result of the axiom (0-4) we know that $p B$ can be described as those functions in $B$ vanishing to the required multiplicities on $Z(J)$. Note that our axiom (0-2) (the continuity of point evaluation at each $\lambda \in \Omega$ ) implies that $p B$ is closed in $B$. It is easily seen that $p B$ is a multiplier invariant subspace of $B$ containing $J$. We claim that $J=p B$. Put $J / p=J_{1}$. It is clear that $J_{1}$ is a multiplier invariant subspace of $B$, and that $J=p J_{1} \subset J_{1}$. We first note that our assumption on $\sigma_{J}$ entails that $J$ has index one (see Corollary 2.4), and secondly if we temporarily accept that

$$
\sigma_{J_{1}}=\sigma\left(M_{z}\left[B / J_{1}\right]\right) \subset \sigma\left(M_{z}[B / J]\right) \subset \Omega,
$$

then using the fact that $Z\left(J_{1}\right)=\emptyset$ we conclude that $\sigma_{J_{1}}=Z\left(J_{1}\right) \cap \Omega=\emptyset$. Therefore $B / J_{1}=\{0\}$, or equivalently $J_{1}=B$ and finally $J=p J_{1}=p B$. Now, the only thing which requires verification is $\sigma_{J_{1}} \subset \sigma_{J}$. To this end, define $\Psi: p B / J \rightarrow B / J_{1}$ by $p f+J \mapsto f+J_{1}$, for $f \in B$. First note that for $f$ and $g$ in $B$, the equality $p f+J=p g+J$ is equivalent to $(f-g) \in J / p=J_{1}$, or $f+J_{1}=g+J_{1}$, so that the mapping $\Psi$ is well-defined and one-to-one. The surjectivity of $\Psi$ is also clear, making it an isomorphism. Now, assume that $T_{1}$ and $T_{2}$ are the operators of multiplication by $z$ on $B / J_{1}$ and $p B / J$, respectively. We then have

$$
\begin{aligned}
\Psi\left(T_{2}(p f+J)\right)=\Psi(z p f+J)=z f+J_{1} & =T_{1}\left(f+J_{1}\right) \\
& =T_{1}(\Psi(p f+J)) .
\end{aligned}
$$

Therefore $\Psi T_{2}=T_{1} \Psi$, or equivalently $T_{2}=\Psi^{-1} T_{1} \Psi$. Similarly, we can obtain $\lambda-T_{2}=\Psi^{-1}\left(\lambda-T_{1}\right) \Psi$ for every $\lambda \in \mathbb{C}$, which in turn shows 
that $\sigma\left(T_{1}\right)=\sigma\left(T_{2}\right)$. Now, let $\lambda \in \mathbb{C} \backslash \sigma_{J}$, and define the operator $A_{\lambda}: p B / J \rightarrow p B / J$ by the following:

$$
A_{\lambda}(p f+J)=p\left(M_{z}[B / J]\right)\left(\lambda-M_{z}[B / J]\right)^{-1}(f+J), \quad f \in B .
$$

We first note that for every natural number $n$, and every $f \in B$ we have

$$
\left(M_{z}[B / J]\right)^{n}(f+J)=\left(M_{z}[B / J]\right)^{n-1}(z f+J),
$$

so that

$$
\left(M_{z}[B / J]\right)^{n}(f+J)=z^{n} f+J=M_{z^{n}}[B / J](f+J) .
$$

Therefore $p\left(M_{z}[B / J]\right)=M_{p}[B / J]$ takes $B / J$ onto $p B / J$. A straightforward calculation shows that for $\lambda \in \mathbb{C} \backslash \bar{\Omega}$, one has

$$
\left(\lambda-M_{z}[p B / J]\right) A_{\lambda}(f+J)=f+J,
$$

that is, $\left(\lambda-M_{z}[p B / J]\right) A_{\lambda}$ equals the identity operator for every $\lambda \in$ $\mathbb{C} \backslash \bar{\Omega}$. It is easy to see that for such $\lambda$, the operator $A_{\lambda}$ commutes with $\lambda-M_{z}[p B / J]$. But $A_{\lambda}$ is analytic in the $\lambda$ variable in $\mathbb{C} \backslash \sigma_{J}$, and also $\lambda-M_{z}[p B / J]$ is an entire function of $\lambda$, therefore their composition is analytic in $\mathbb{C} \backslash \sigma_{J}$. It follows from the uniqueness theorem for vector valued analytic functions that in fact $\left(\lambda-M_{z}[B / J]\right) A_{\lambda}$ equals the identity operator for all $\lambda \in \mathbb{C} \backslash \sigma_{J}$, which means that $A_{\lambda}$ is the inverse operator to $\lambda-M_{z}[p B / J]$ for all $\lambda \in \mathbb{C} \backslash \sigma_{J}$, and hence

$$
\sigma_{J_{1}}=\sigma\left(T_{1}\right)=\sigma\left(T_{2}\right)=\sigma\left(M_{z}[p B / J]\right) \subset \sigma_{J} .
$$

The proof is complete.

\section{The main result}

This section aims to carry out the following plan. Suppose $\Omega$ is a bounded planar domain and $B$ a Banach space of analytic functions on $\Omega$ which contains 1 and satisfies the axioms (0-1)-(0-4). Let $B_{1}$ be a closed subspace of $B$ containing both the constant function 1 and the coordinate function $z$, and let $I$ be a proper multiplier invariant subspace of $B_{1}$. Consider the mapping $H_{I}: \mathcal{O}\left(\sigma\left(M_{z}\left[B_{1} / I\right]\right)\right) \rightarrow \mathcal{L}\left(B_{1} / I\right)$ defined by

$$
f \mapsto \frac{1}{2 \pi i} \int_{\partial U} f(\lambda)\left(\lambda-M_{z}\left[B_{1} / I\right]\right)^{-1} d \lambda,
$$


where $U$ is some finitely connected smoothly bordered domain surrounding the spectrum $\sigma\left(M_{z}\left[B_{1} / I\right]\right)$ such that $\bar{U}$ lies in the domain of analyticity of $f$, and $\partial U$ is oriented in the positive direction. Note that by Cauchy formula the above integral does not depend on the choice of $U$, moreover, if $\mathcal{O}\left(\sigma\left(M_{z}\left[B_{1} / I\right]\right)\right)$, the algebra of all analytic functions on $\sigma\left(M_{z}\left[B_{1} / I\right]\right)$, is endowed with compact open topology, then the above mapping is continuous. Aside from these properties, this mapping has the following specifications:

(1) It is a homomorphism, because it is multiplicative, and

(2) It maps unit to unit, and $z$ to $M_{z}\left[B_{1} / I\right]$.

Therefore, with some abuse of notation, we have a module-homomorphism $L_{I}$, from $\mathcal{O}\left(\sigma\left(M_{z}\left[B_{1} / I\right]\right)\right)$ into $B_{1} / I$ which takes unit to unit and $z$ to $z+I$. We recall that the action of $L_{I}$ on an element $h \in$ $\mathcal{O}\left(\sigma\left(M_{z}\left[B_{1} / I\right]\right)\right)$ is defined to be the action of $H_{I}(h)$ on the element $1+I$ of $B_{1}$. We shall at times refer to this mapping as HFC for the involvement of the holomorphic functional calculus. However, we notice that this mapping is in fact $L_{I}=P_{I} \circ H_{I}$ as defined earlier. One of the most important features of HFC as defined above is its compatibility in the following manner: If $B=B_{1} \oplus B_{2}^{0}$ then the canonical surjective modulehomomorphism $B_{1} \rightarrow B_{1} / I$, the module-homomorphism which sends $f$ to $f+I$, and the HFC module-homomorphism are compatible in such a way that they define a module-homomorphism $L_{I}: B \rightarrow B_{1} / I$. Furthermore, the quotient Banach spaces $B_{1} / I$ and $B /$ ker $L_{I}$ are canonically module-isomorphic; this means that $\overline{L_{I}}: B / \operatorname{ker} L_{I} \rightarrow B_{1} / I$ is injective and surjective, furthermore, its inverse is also a module-homomorphism. It is desirable to reveal the relationship between $I$ and $\operatorname{ker} L_{I}$. The objective of this paper is achieved whenever this is done for a reasonable class of $M\left(B_{1}\right)$-invariant subspaces.

For a bounded planar domain $\Omega$, we can find two other planar domains $\Omega_{1}$ and $\Omega_{2}$ such that:

(1) $\Omega_{1}$ is bounded,

(2) $\Omega_{2}$ is unbounded and non-dense in the complex plane,

(3) $\Omega=\Omega_{1} \cap \Omega_{2}$, and $\Omega_{1} \cup \Omega_{2}=\mathbb{C}$.

Define the projection $P_{1}: \mathcal{O}(\Omega) \rightarrow \mathcal{O}\left(\Omega_{1}\right)$ by the following formula

$$
P_{1} f(z)=\frac{1}{2 \pi i} \int_{\partial V_{n}}(\zeta-z)^{-1} f(\zeta) d \zeta \quad z \in V_{n},
$$


where $\left\{V_{n}\right\}_{n=1}^{\infty}$ is an increasing sequence of finitely connected smoothly bordered subdomains of $\Omega_{1}$ satisfying the relations $V_{1} \supset \mathbb{C} \backslash \Omega_{2}$ and $\cup_{n=1}^{\infty} V_{n}=\Omega_{1}$. As usual in the theory of one complex variables, the boundary of $V_{n}$ is oriented in the positive direction. The above formula does not depend on the choice of $V_{n}$, and furnishes us with a continuous projection $P_{1}: \mathcal{O}(\Omega) \rightarrow \mathcal{O}\left(\Omega_{1}\right)$. Similarly one can find a continuous projection $P_{2}: \mathcal{O}(\Omega) \rightarrow \mathcal{O}_{0}\left(\Omega_{2}\right)$ with the condition that $P_{1}+P_{2}=$ identity. Note that by Liouville's theorem both $P_{1}$ and $P_{2}$ are uniquely determined. We also note that $P_{1}$ and $P_{2}$ map $B$ into $\mathcal{O}\left(\Omega_{1}\right)$ and $\mathcal{O}_{0}\left(\Omega_{2}\right)$, respectively. Keeping in mind that $B$ is a Banach space of analytic functions on $\Omega$ which contains 1 and satisfies the axioms (0-1)-(0-4), we put $B_{1}=B \cap \mathcal{O}\left(\Omega_{1}\right), B_{2}=B \cap \mathcal{O}\left(\Omega_{2}\right)$, and $B_{2}^{0}=B \cap \mathcal{O}_{0}\left(\Omega_{2}\right)$. It is evident that $B_{1}$ and $B_{2}^{0}$ are two closed subspaces of $B$. Let $f \in B_{1} \cap B_{2}^{0}$, then $f \in \mathcal{O}(\mathbb{C})$ and $f(z) \rightarrow 0$ as $|z| \rightarrow \infty$; so that by Liouville's theorem, $f$ is identically 0 . It is also obvious that $B \supset B_{1} \oplus B_{2}^{0}$, the direct sum of $B_{1}$ and $B_{2}^{0}$. We shall assume that $B$ can be written as the direct sum of $B_{1}$ and $B_{2}^{0}$, and that $B_{1}$ and $B_{2}^{0}$ satisfy the axioms (0-1)-(0-4) on $\Omega_{1}$ and $\Omega_{2}$, respectively. We emphasize that $B_{1}$ and $B_{2}^{0}$ automatically satisfy the first three axioms. Furthermore, $B_{1}$ satisfies the fourth axiom for every $\lambda \in \Omega_{1}$ except those on the boundary of $\Omega_{2}$. The same is true for $B_{2}^{0}$ also. Note that our assumption that $B=B_{1} \oplus B_{2}^{0}$ is equivalent to either of $P_{1} B \subset B$ or $P_{2} B \subset B$.

Now, suppose that $I$ is a proper multiplier invariant subspace of $B_{1}$ and recall the HFC module-homomorphism $\mathcal{O}\left(\sigma\left(M_{z}\left[B_{1} / I\right]\right)\right) \rightarrow B_{1} / I$ given by

$$
f \mapsto\left(\frac{1}{2 \pi i} \int_{\partial U} f(\lambda)\left(\lambda-M_{z}\left[B_{1} / I\right]\right)^{-1} d \lambda\right)(1+I),
$$

where $U$ is some finitely connected smoothly bordered domain surrounding the spectrum of the operator $M_{z}\left[B_{1} / I\right]: B_{1} / I \rightarrow B_{1} / I$. If we assume that $\sigma\left(M_{z}\left[B_{1} / I\right]\right) \subset \Omega_{2}$, then HFC module-homomorphism furnishes us with a continuous module-homomorphism $B_{2}^{0} \rightarrow B_{1} / I$, because of

$$
B_{2}^{0} \subset \mathcal{O}_{0}\left(\Omega_{2}\right) \subset \mathcal{O}\left(\sigma\left(M_{z}\left[B_{1} / I\right]\right)\right) .
$$

Now we are in a place which allows us to construct our desired surjective module-homomorphism which we again write

$$
L_{I}: B=B_{1} \oplus B_{2}^{0} \rightarrow B_{1} / I,
$$

defined to be the canonical module-homomorphism on $B_{1}$, that is, $L_{I}(f)=f+I$ for $f \in B_{1}$ and the HFC module-homomorphism on $B_{2}^{0}$. The following lemma tells us more about $L_{I}$. 
Before proceeding, let us fix once again our assumptions on $B$. We know that $B$ is a Banach space of analytic functions defined on a bounded domain $\Omega$ in the extended complex plane. Furthermore, $B$ contains 1 , satisfies the axioms (0-1)-(0-4), and has $B=B_{1} \oplus B_{2}^{0}$. We shall also make the assumption that $M(B)=M\left(B_{1}\right) \oplus M_{0}\left(B_{2}\right)$, where $M_{0}\left(B_{2}\right)=$ $M\left(B_{2}\right) \cap \mathcal{O}_{0}\left(\Omega_{2}\right)$.

Lemma 3.1. Let $I$ be an $M\left(B_{1}\right)$-invariant subspace of $B_{1}$ satisfying $\sigma\left(M_{z}\left[B_{1} / I\right]\right) \subset \Omega_{2}$. If $\mathcal{O}_{0}\left(\bar{\Omega}_{2}\right)$ is dense in $M_{0}\left(B_{2}\right)$ and in $B_{2}^{0}$, then $L_{I}$ is a surjective module-homomorphism $B=B_{1} \oplus B_{2}^{0} \rightarrow B_{1} / I$.

Proof: The surjectivity of $L_{I}$ is obvious and the continuity is a consequence of the closed graph theorem. We just need to verify that for every $\varphi \in M(B)$ and every $f \in B$, the following equality holds

$$
L_{I}(\varphi f)=L_{I, M}(\varphi) L_{I}(f),
$$

where $L_{I, M}: M(B) \rightarrow \mathcal{L}\left(B_{1} / I\right)$ is an appropriate continuous homomorphism. Since $L_{I}$ is onto, it follows from Remark 1.2 that there exists at most one such homomorphism. Let $\varphi \in M(B)$ be written as $\varphi=\varphi_{1}+\varphi_{2}$ where $\varphi_{1} \in M\left(B_{1}\right)$ and $\varphi_{2} \in M_{0}\left(B_{2}\right)$. Put

$$
L_{I, M}(\varphi)=L_{I, M}\left(\varphi_{1}+\varphi_{2}\right)=M_{\varphi_{1}}\left[B_{1} / I\right]+\varphi_{2}\left(M_{z}\left[B_{1} / I\right]\right) .
$$

Here, $M_{\varphi_{1}}\left[B_{1} / I\right]$ stands for the operator on $B_{1} / I$ which sends $f+I$ to $\varphi f+I$. Note that the second operator in the definition of $L_{I, M}$ comes from the holomorphic functional calculus. It is not difficult to see that $L_{I, M}$ is a homomorphism; the important fact which requires verification is to show that for $\varphi_{1} \in M\left(B_{1}\right)$ and $\varphi_{2} \in M_{0}\left(B_{2}\right)$, we have

$$
L_{I, M}\left(\varphi_{1} \varphi_{2}\right)=L_{I, M}\left(\varphi_{1}\right) L_{I, M}\left(\varphi_{2}\right)=L_{I, M}\left(\varphi_{2}\right) L_{I, M}\left(\varphi_{1}\right) .
$$

We first check this for $\varphi_{2}(z)=(\lambda-z)^{-1}$, with $\lambda \in \mathbb{C} \backslash \bar{\Omega}_{2}$. In this case

$$
\frac{\varphi_{1}(z)}{\lambda-z}=\frac{\varphi_{1}(z)-\varphi_{1}(\lambda)}{\lambda-z}+\frac{\varphi_{1}(\lambda)}{\lambda-z} \text {. }
$$

Note that the first term on the right hand side of (3-1) is an element of $M\left(B_{1}\right)$. To see this, put $T_{\lambda} \varphi_{1}(z)=\frac{\varphi_{1}(\lambda)-\varphi_{1}(z)}{\lambda-z}$, and let $\lambda \in \Omega_{1}, f \in B_{1}$. We first note that by axiom (0-4), $T_{\lambda} \varphi_{1} \in B_{1}$. We then have

$$
\begin{aligned}
T_{\lambda} \varphi_{1}(z) \cdot f(z) & =\frac{\varphi_{1}(\lambda)-\varphi_{1}(z)}{\lambda-z} \cdot f(z) \\
& =T_{\lambda}\left(\varphi_{1}(z) f(z)\right)+\frac{\varphi_{1}(\lambda) f(z)-\varphi_{1}(\lambda) f(\lambda)}{\lambda-z} \\
& =T_{\lambda}\left(\varphi_{1}(z) f(z)\right)-\varphi_{1}(\lambda) T_{\lambda} f(z) .
\end{aligned}
$$


We now use the axiom (0-4) to get $T_{\lambda} \varphi_{1}(z) \in M\left(B_{1}\right)$. We also note that $\varphi_{1}(\lambda)(\lambda-z)^{-1} \in M_{0}\left(B_{2}\right)$, because it is a rational function with pole outside $\bar{\Omega}_{2}$. Hence, by $(3-1)$,

$$
L_{I, M}\left(\varphi_{1} \cdot(\lambda-z)^{-1}\right)=M_{T_{\lambda} \varphi_{1}}\left[B_{1} / I\right]+\varphi_{1}(\lambda)\left(\lambda-M_{z}\left[B_{1} / I\right]\right)^{-1} .
$$

We claim that the right hand side is equal to $M_{\varphi_{1}}\left[B_{1} / I\right]\left(\lambda-M_{z}\left[B_{1} / I\right]\right)^{-1}$ and to $\left(\lambda-M_{z}\left[B_{1} / I\right]\right)^{-1} M_{\varphi_{1}}\left[B_{1} / I\right]$. To prove the claim, we multiply both sides by $\left(\lambda-M_{z}\left[B_{1} / I\right]\right)=M_{\lambda-z}\left[B_{1} / I\right]$, from the right in one case and from the left in the other. So that the question is whether

$$
M_{T_{\lambda} \varphi_{1}}\left[B_{1} / I\right]\left(\lambda-M_{z}\left[B_{1} / I\right]\right)+\varphi_{1}(\lambda)=M_{\varphi_{1}}\left[B_{1} / I\right] .
$$

Since in general, for $\varphi$ and $\psi$ in $M\left(B_{1}\right)$ we have $M_{\varphi}\left[B_{1} / I\right] M_{\psi}\left[B_{1} / I\right]=$ $M_{\varphi \psi}\left[B_{1} / I\right]$, the question transforms into whether $M_{\varphi_{1}(z)-\varphi_{1}(\lambda)}\left[B_{1} / I\right]+$ $\varphi_{1}(\lambda)=M_{\varphi_{1}(z)}\left[B_{1} / I\right]$, which is true. Therefore the claim is proved, and consequently

$$
L_{I, M}\left(\varphi_{1} \cdot(\lambda-z)^{-1}\right)=L_{I, M}\left(\varphi_{1}\right) L_{I, M}\left((\lambda-z)^{-1}\right)
$$

Since finite linear combinations of $z \mapsto(\lambda-z)^{-1}$ for $\lambda \in \mathbb{C} \backslash \bar{\Omega}_{2}$ are dense in $\mathcal{O}_{0}\left(\bar{\Omega}_{2}\right)$, we obtain

$$
\begin{aligned}
& L_{I, M}\left(\varphi_{1} \varphi_{2}\right)=L_{I, M}\left(\varphi_{1}\right) L_{I, M}\left(\varphi_{2}\right) \\
& \qquad \text { for } \varphi_{1} \in M\left(B_{1}\right) \text { and } \varphi_{2} \in \mathcal{O}_{0}\left(\bar{\Omega}_{2}\right)
\end{aligned}
$$

Finally because of our assumption that $\mathcal{O}_{0}\left(\bar{\Omega}_{2}\right)$ is dense in $M_{0}\left(B_{2}\right)$, the equality (3-3) holds for every $\varphi_{1} \in M\left(B_{1}\right)$ and every $\varphi_{2} \in M_{0}\left(B_{2}\right)$. We now turn to prove that $L_{I}$ is a module-homomorphism. To do this, we consider four cases.

Case 1: $\varphi \in M\left(B_{1}\right)$ and $f \in B_{1}$; we have $\varphi f \in B_{1}$, therefore

$$
L_{I}(\varphi f)=\varphi f+I=M_{\varphi}\left[B_{1} / I\right](f+I)=L_{I, M}(\varphi) L_{I}(f) .
$$

Case 2: $\varphi \in M\left(B_{1}\right)$ and $f \in B_{2}^{0}$; it suffices to treat the case when $f=(\lambda-z)^{-1}$ for $\lambda \in \mathbb{C} \backslash \bar{\Omega}_{2}$, because $\mathcal{O}_{0}\left(\bar{\Omega}_{2}\right)$ is dense in $B_{2}^{0}$. We then have the decomposition

$$
\varphi f=\frac{\varphi}{\lambda-z}=\frac{\varphi-\varphi(\lambda)}{\lambda-z}+\frac{\varphi(\lambda)}{\lambda-z}
$$


where the first term is in $M\left(B_{1}\right) \subset B_{1}$ (see above) and the second one in $M_{0}\left(B_{2}\right) \subset B_{2}^{0}$. Hence

$$
\begin{aligned}
L_{I}(\varphi f) & =\left(T_{\lambda} \varphi+I\right)+\varphi(\lambda)\left(\lambda-M_{z}\left[B_{1} / I\right]\right)^{-1}(1+I) \\
& =M_{T_{\lambda} \varphi}(1+I)+\varphi(\lambda)\left(\lambda-M_{z}\left[B_{1} / I\right]\right)^{-1}(1+I) \\
& =\left[M_{T_{\lambda} \varphi}+\varphi(\lambda)\left(\lambda-M_{z}\left[B_{1} / I\right]\right)^{-1}\right](1+I) .
\end{aligned}
$$

We now use (3-2) to obtain

$$
L_{I}(\varphi f)=M_{\varphi}\left[B_{1} / I\right]\left(\lambda-M_{z}\left[B_{1} / I\right]\right)^{-1}(1+I)=L_{I, M}(\varphi) L_{I}(f) .
$$

Case 3: $\varphi \in M_{0}\left(B_{2}\right)$ and $f \in B_{2}^{0}$; the desired equality is a consequence of the holomorphic functional calculus, since $\varphi f \in B_{2}^{0}$.

Case 4: $\varphi \in M_{0}\left(B_{2}\right)$ and $f \in B_{1}$; we may assume that $\varphi(z)=(\lambda-z)^{-1}$ for $\lambda \in \mathbb{C} \backslash \bar{\Omega}_{2}$, because $\mathcal{O}_{0}\left(\bar{\Omega}_{2}\right)$ is dense in $M_{0}\left(B_{2}\right)$. This time we use the following decomposition of $\varphi f$;

$$
\varphi f=\frac{f(z)-f(\lambda)}{\lambda-z}+\frac{f(\lambda)}{\lambda-z}=-T_{\lambda} f(z)+\frac{f(\lambda)}{\lambda-z} .
$$

By the axiom (0-4), the first term is in $B_{1}$ and the second one is in $M_{0}\left(B_{2}\right) \subset B_{2}^{0}$. For $\lambda \in \Omega_{1}$, it is clear that $T_{\lambda} \in \mathcal{L}\left(B_{1}\right)$, which associates to a given $f \in B_{1}$, the element $T_{\lambda} f \in B_{1}$. There is an associated operator $T_{\lambda}\left[B_{1} / I\right]$ on $B_{1} / I$, which maps a given $f+I \in B_{1} / I$ onto the element $T_{\lambda} f+I \in B_{1} / I$. Therefore, on $B_{1}$ we have $\left(\lambda-M_{z}\right) T_{\lambda} f=f(\lambda)-f$, and on $B_{1} / I$ we have

$$
\left(\left(\lambda-M_{z}\left[B_{1} / I\right]\right) T_{\lambda}\left[B_{1} / I\right]\right) f=f(\lambda)-f+I,
$$

or equivalently

$$
T_{\lambda}\left[B_{1} / I\right] f=\left(\lambda-M_{z}\left[B_{1} / I\right]\right)^{-1}(f(\lambda)-f+I) .
$$

Finally, from (3-4) and (3-5) we have

$$
\begin{aligned}
L_{I}(\varphi f) & =-T_{\lambda}\left[B_{1} / I\right] f+f(\lambda)\left(\lambda-M_{z}\left[B_{1} / I\right]\right)^{-1}(1+I) \\
& =\left(\lambda-M_{z}\left[B_{1} / I\right]\right)^{-1}(f-f(\lambda)+I)+\left(\lambda-M_{z}\left[B_{1} / I\right]\right)^{-1}(f(\lambda)+I) \\
& =\left(\lambda-M_{z}\left[B_{1} / I\right]\right)^{-1}(f+I) \\
& =L_{I, M}(\varphi) L_{I}(f) .
\end{aligned}
$$

The proof is now complete. 
In the proof of Lemma 3.1, we used the assumptions that $\mathcal{O}_{0}\left(\bar{\Omega}_{2}\right)$ is dense in both $M_{0}\left(B_{2}\right)$ and $B_{2}^{0}$. In the following, we shall try to get rid of these assumptions. Let $\Omega_{2, \sharp}$ be a finitely connected smoothly bordered domain such that

$$
\left(\mathbb{C} \backslash \Omega_{1}\right) \cup \sigma\left(M_{z}\left[B_{1} / I\right]\right) \subset \Omega_{2, \sharp} \subset \bar{\Omega}_{2, \sharp} \subset \Omega_{2} .
$$

Lemma 3.2. Let $I$ be an $M\left(B_{1}\right)$-invariant subspace of $B_{1}$ satisfying $\sigma\left(M_{z}\left[B_{1} / I\right]\right) \subset \Omega_{2}$. Then there exists a pair $\left(L_{I}, L_{I, M}\right)$ with the following properties:

$L_{I}: B_{1} \oplus \mathcal{O}_{0}\left(\bar{\Omega}_{2, \sharp}\right) \rightarrow B_{1} / I$ and $L_{I, M}: M\left(B_{1}\right) \oplus \mathcal{O}_{0}\left(\bar{\Omega}_{2, \sharp}\right) \rightarrow \mathcal{L}\left(B_{1} / I\right)$, such that $L_{I}(\varphi f)=L_{I, M}(\varphi) L_{I}(f)$ for every $\varphi \in M\left(B_{1}\right) \oplus \mathcal{O}_{0}\left(\bar{\Omega}_{2, \sharp}\right)$ and every $f \in B_{1} \oplus \mathcal{O}_{0}\left(\bar{\Omega}_{2, \sharp}\right)$, and such that $L_{I, M}\left(\varphi_{1} \varphi_{2}\right)=L_{I, M}\left(\varphi_{1}\right) L_{I, M}\left(\varphi_{2}\right)$ for every $\varphi_{1}$ and $\varphi_{2}$ in $M\left(B_{1}\right) \oplus \mathcal{O}_{0}\left(\bar{\Omega}_{2, \sharp}\right)$.

Proof: The proof is exactly as that of Lemma 3.1, if we notice that finite linear combinations of rational functions of the form $(\lambda-z)^{-1}$ for $\lambda \in \mathbb{C} \backslash \bar{\Omega}_{2, \sharp}$ are dense in $\mathcal{O}_{0}\left(\bar{\Omega}_{2, \sharp}\right)$, and that $M\left(B_{1}\right) \oplus \mathcal{O}_{0}\left(\bar{\Omega}_{2, \sharp}\right)$ is an algebra of analytic functions. To this end, it suffices to verify that for every $\varphi \in M\left(B_{1}\right)$ and every $f \in \mathcal{O}_{0}\left(\bar{\Omega}_{2, \sharp}\right)$ we have $\varphi f \in M\left(B_{1}\right) \oplus$ $\mathcal{O}_{0}\left(\bar{\Omega}_{2, \sharp}\right)$. We may assume that $f(z)=(\lambda-z)^{-1}$ for $\lambda \in \mathbb{C} \backslash \bar{\Omega}_{2, \sharp}$. Let us first write

$$
\varphi(z) f(z)=-T_{\lambda} \varphi(z)+\frac{\varphi(\lambda)}{\lambda-z}
$$

We have observed in the proof of Lemma 3.1 that $-T_{\lambda} \varphi(z) \in M\left(B_{1}\right)$, hence $\varphi(z) f(z) \in M\left(B_{1}\right) \oplus \mathcal{O}_{0}\left(\bar{\Omega}_{2, \sharp}\right)$. The last thing which requires verification is the following

$$
L_{I, M}\left(\varphi_{1} \varphi_{2}\right)=L_{I, M}\left(\varphi_{1}\right) L_{I, M}\left(\varphi_{2}\right), \quad \varphi_{1} \in M\left(B_{1}\right), \varphi_{2} \in \mathcal{O}_{0}\left(\bar{\Omega}_{2, \sharp}\right) .
$$

Since $\varphi_{2}$ is analytic in a neighbourhood of $\bar{\Omega}_{2, \sharp}$, we can then apply the Cauchy integral formula to get

$$
\varphi_{2}(z)=\frac{1}{2 \pi i} \int_{\partial U} \varphi_{2}(\lambda)(\lambda-z)^{-1} d \lambda, \quad z \in \Omega_{2, \sharp},
$$

where $U$ is some smoothly bordered domain containing $\bar{\Omega}_{2, \sharp}$, where $\varphi_{2}$ is analytic. Now we have

$$
\varphi_{1}(z) \varphi_{2}(z)=\frac{1}{2 \pi i} \int_{\partial U} \varphi_{2}(\lambda) \varphi_{1}(z)(\lambda-z)^{-1} d \lambda \quad z \in \Omega_{1} \cap \Omega_{2, \sharp},
$$


and finally

$$
\begin{aligned}
L_{I, M}\left(\varphi_{1} \varphi_{2}\right) & =\frac{1}{2 \pi i} \int_{\partial U} \varphi_{2}(\lambda) L_{I, M}\left(\varphi_{1}\right) L_{I, M}\left((\lambda-z)^{-1}\right) d \lambda \\
& =L_{I, M}\left(\varphi_{1}\right)\left(\frac{1}{2 \pi i} \int_{\partial U} \varphi_{2}(\lambda)\left(\lambda-M_{z}\left[B_{1} / I\right]\right)^{-1} d \lambda\right) \\
& =L_{I, M}\left(\varphi_{1}\right) L_{I, M}\left(\varphi_{2}\right) .
\end{aligned}
$$

The proof is complete.

Restricting to $B=B_{1} \oplus B_{2}^{0}$, we obtain the following consequence.

Proposition 3.3. Let $I$ be an $M\left(B_{1}\right)$-invariant subspace of $B_{1}$ satisfying the condition $\sigma\left(M_{z}\left[B_{1} / I\right]\right) \subset \Omega_{2}$ and let $L_{I}$ be as in Lemma 3.2. Consider the restriction $L_{I}: B=B_{1} \oplus B_{2}^{0} \rightarrow B_{1} / I$. Then $L_{I}$ is a module-homomorphism.

Proof: We need to note that such an $\Omega_{2, \sharp}$ satisfying (3-6) always exists, and that $B=B_{1} \oplus B_{2}^{0} \subset B_{1} \oplus \mathcal{O}_{0}\left(\bar{\Omega}_{2, \sharp}\right)$, and finally that $M(B)=M\left(B_{1}\right) \oplus M_{0}\left(B_{2}\right) \subset M\left(B_{1}\right) \oplus \mathcal{O}_{0}\left(\bar{\Omega}_{2, \sharp}\right)$.

Proposition 3.4. Let $I$ be an $M\left(B_{1}\right)$-invariant subspace of $B_{1}$ satisfying the condition $\sigma\left(M_{z}\left[B_{1} / I\right]\right) \subset \Omega_{2}$ and let $L_{I}$ be as in Proposition 3.3. If $J=\operatorname{ker} L_{I}$, then

(a) $\cos (I \cdot M(B)) \subset J$,

(b) $J \cap B_{1}=I$, and

(c) $\sigma\left(M_{z}\left[B_{1} / I\right]\right)=\sigma\left(M_{z}[B / J]\right)$.

Proof: For ease of notation, we put $\Lambda(I)=\operatorname{clos}(I \cdot M(B))$. Suppose that $f \in \Lambda(I)$, then there exists a sequence $f_{n} \rightarrow f, f_{n} \in I \cdot M(B)$. Put $f_{n}=\sum_{k=1}^{m} h_{n, k} g_{n, k}$ where $h_{n, k} \in M(B)$ and $g_{n, k} \in I$. Now, we have

$$
L_{I}\left(f_{n}\right)=\sum_{k=1}^{m} L_{I, M}\left(h_{n, k}\right) L_{I}\left(g_{n, k}\right)=\sum_{k=1}^{m} L_{I, M}\left(h_{n, k}\right) 0=0,
$$

which proves $L_{I}(f)=0$, by continuity. This shows that $\Lambda(I) \subset J$.

To prove part (b), we first note that the inclusion $I \subset J \cap B_{1}$ is obvious. For the reverse inclusion, let $f \in J \cap B_{1}$, then from the definitions of $L_{I}$ and $J$ we have $L_{I}(f)=f+I=0+I$ or equivalently $f \in I$, hence $J \cap B_{1} \subset I$ and finally

$$
J \cap B_{1}=\left(\operatorname{ker} L_{I}\right) \cap B_{1}=I .
$$


The last thing to be proved is the equality

$$
\sigma\left(M_{z}[B / J]\right)=\sigma\left(M_{z}\left[B_{1} / I\right]\right),
$$

for all $M\left(B_{1}\right)$-invariant subspaces $I$ with $\sigma\left(M_{z}\left[B_{1} / I\right]\right) \subset \Omega_{2}$. To prove this, we first recall the connection between the mapping $L_{I}: B \rightarrow B_{1} / I$ and the operator $M_{z}\left[B_{1} / I\right]$ defined on $B_{1} / I$. It is immediate that for every $f \in B_{1}$ we have $L_{I}(z f)=M_{z}\left[B_{1} / I\right] L_{I}(f)$. Moreover, if $f \in B_{2}^{0}$ then $L_{I}=P_{I} \circ H_{I}$ (see section 1) and we have

$$
\begin{aligned}
L_{I}(z f)=P_{I}\left(H_{I}(z f)\right)=P_{I}\left(H_{I}(z) H_{I}(f)\right) & =H_{I}(z) H_{I}(f)(1+I) \\
& =M_{z}\left[B_{1} / I\right] L_{I}(f) .
\end{aligned}
$$

Therefore the equality $L_{I}(z f)=M_{z}\left[B_{1} / I\right] L_{I}(f)$ holds for every $f \in B$. We know that $J=\operatorname{ker} L_{I}$, therefore the surjective module-homomorphism $L_{I}$ induces an isomorphism $\overline{L_{I}}: B / J \rightarrow B_{1} / I$ defined by $\bar{L}_{I}(f+J)=L_{I}(f)$, hence

$$
\begin{aligned}
\overline{L_{I}}\left(M_{z}[B / J](f+J)\right)=\overline{L_{I}}(z f+J)=L_{I}(z f) & =M_{z}\left[B_{1} / I\right] L_{I}(f) \\
& =M_{z}\left[B_{1} / I\right] \overline{L_{I}}(f+J) .
\end{aligned}
$$

So that $\overline{L_{I}}$ carries over $M_{z}[B / J]$ to $M_{z}\left[B_{1} / I\right]$; more precisely we have $\overline{L_{I}} M_{z}[B / J]=M_{z}\left[B_{1} / I\right] \overline{L_{I}}$. Now it is easily seen that if $\left(\lambda-M_{z}\left[B_{1} / I\right]\right)^{-1}$ exists for some complex number $\lambda$, then $\left(\lambda-M_{z}[B / J]\right)^{-1}$ exists too. In fact we have the following equality

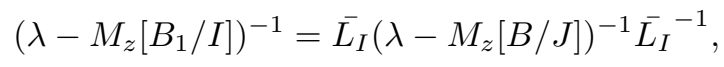

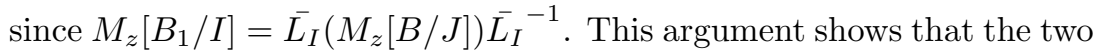
spectra are the same, hence the proof is complete.

As we shall see later on, Proposition 3.4 will constitute a considerable part of our main result, Theorem 3.10. What we intend to show is equality in part (a), instead of inclusion. Let us temporarily assume that we have been able to demonstrate the equality in part (a). Thus, we would have a mapping, say $\Lambda$, from the collection of all $M\left(B_{1}\right)$-invariant subspaces $I$ with $\sigma\left(M_{z}\left[B_{1} / I\right]\right) \subset \Omega_{2}$ into the collection of all $M(B)$-invariant subspaces $J$ with the same condition on the spectrum $\sigma\left(M_{z}[B / J]\right)$. The image of $I$ under the mapping $\Lambda$ is defined to be

$$
\Lambda(I)=\operatorname{clos}(I \cdot M(B))=\operatorname{ker} L_{I}=J .
$$


Part (b) of the above proposition tells us that the mapping $\Lambda$ is injective. Now, assume that $J$ is an $M(B)$-invariant subspace of $B$ satisfying $\sigma\left(M_{z}[B / J]\right) \subset \Omega_{2}$. Concerning the surjectivity of $\Lambda$, we will look at $J \cap B_{1}$ as a natural candidate, as part (b) of the above proposition suggests. Unfortunately, it might happen that $J \cap B_{1}$ collapses to the trivial null subspace, which in turn will destroy the equality $\Lambda\left(J \cap B_{1}\right)=J$. To prevent this event, we follow Hedenmalm [16] and make the assumption that the Banach space $B$ of analytic functions on $\Omega$ satisfies the shrinking domain condition (see Definition 3.5). This assumption makes it possible to prove a factorization theorem which turns out to be crucial both to the proof of the equality in part (a) of Proposition 3.4 and to the proof of the surjectivity of the mapping $\Lambda$. Given $f \in B$, it is not difficult to find two functions $f_{1}$ and $f_{2}$, analytic in $\Omega_{1}$ and $\Omega_{2}$ respectively, such that $f=f_{1} \cdot f_{2}$. But, in general, there is no guarantee that $f_{1} \in B_{1}$ and $f_{2} \in B_{2}$. The "shrinking domain" condition which is easy to check seems to be what is needed to get the proof of the factorization theorem to go through (see Lemma 3.7). We mention that the authors in [10] used some rather similar arguments to factor out analytic functions in the Dirichlet space.

Let us fix two finitely connected smoothly bordered domains $V_{1}$ and $V_{2}$ such that:

(1) $\mathbb{C} \backslash \Omega_{2} \subset V_{1} \subset \subset \Omega_{1}$,

(2) $\mathbb{C} \backslash \Omega_{1} \subset V_{2} \subset \bar{V}_{2} \subset \Omega_{2}$ and

(3) $\bar{V}_{1} \cap \bar{V}_{2}=\emptyset$.

We now make the following definition.

Definition 3.5. Let $B=B_{1} \oplus B_{2}^{0}$ be a Banach space of analytic functions on $\Omega$ satisfying the axioms (0-1)-(0-4). We say that $B$ satisfies the shrinking domain condition, with respect to the pair $\left(V_{1}, V_{2}\right)$, if there exist a Banach space of analytic functions $B^{(1)}$ on $\Omega_{1} \cap V_{2}$ and a Banach space of analytic functions $B^{(2)}$ on $V_{1} \cap \Omega_{2}$ such that

(a) $B^{(1)}$ and $B^{(2)}$ satisfy the axioms (0-1)-(0-4) with respect to the domains $\Omega_{1} \cap V_{2}$ and $\Omega_{2} \cap V_{1}$, respectively,

(b) $B^{(1)} \cap \mathcal{O}\left(\Omega_{1}\right)=B_{1}$, and $B^{(2)} \cap \mathcal{O}_{0}\left(\Omega_{2}\right)=B_{2}^{0}$,

(c) $B^{(1)}=B_{1} \oplus B_{2,0}^{(1)}$, where $B_{2,0}^{(1)}=B^{(1)} \cap \mathcal{O}_{0}\left(V_{2}\right)$, and $B^{(2)}=$ $B_{1}^{(2)} \oplus B_{2}^{0}$, where $B_{1}^{(2)}=B^{(2)} \cap \mathcal{O}\left(V_{1}\right)$,

(d) $M\left(B^{(1)}\right)=M\left(B_{1}\right) \oplus M_{0}\left(B_{2}^{(1)}\right)$, where $M_{0}\left(B_{2}^{(1)}\right)=M\left(B_{2}^{(1)}\right) \cap$ $\mathcal{O}_{0}\left(V_{2}\right)$, and $M\left(B^{(2)}\right)=M\left(B_{1}^{(2)}\right) \oplus M_{0}\left(B_{2}\right)$. 
Remark 3.6. The conditions of the previous definition are somewhat technical. It is important to realize that in the examples we have mentioned, they are indeed satisfied. For instance, if $B=L_{a}^{2}(\Omega)$, so that $B_{1}=L_{a}^{2}\left(\Omega_{1}\right)$ and $B_{2}=L_{a}^{2}\left(\Omega_{2}\right)$ (here, we use area measure on the Riemann sphere rather than the complex plane to define these Bergman spaces), we can then put $B^{(1)}=L_{a}^{2}\left(\Omega_{1} \cap V_{2}\right)$ and $B^{(2)}=L_{a}^{2}\left(\Omega_{2} \cap V_{1}\right)$. These spaces split as direct summands, and so do the corresponding multiplier spaces, as in this case the multiplier space is $H^{\infty}$ on the respective domain.

Note that by the holomorphic functional calculus, Proposition 1.5(b), we know that

$$
B_{1} \subset \mathcal{O}\left(\Omega_{1}\right) \subset \mathcal{O}\left(\bar{V}_{1}\right) \subset \mathcal{O}\left(\overline{\Omega_{2} \cap V_{1}}\right) \subset B^{(2)} .
$$

On the other hand, $B_{2}^{0} \subset B^{(2)}$, hence $B \subset B^{(2)}$. Similar argument shows that $B \subset B^{(1)}$.

In the sequel we shall need a factorization theorem for a function $f \in B$. To do this, we have to recall the notion of zero set of a function $f \in \mathcal{O}(\Omega)$ where $\Omega$ stands for a planar domain. Define

$$
Z_{\Omega}(f)=\{z \in \Omega: f(z)=0\}, \quad f \in \mathcal{O}(\Omega) .
$$

The following lemma has an important role in the factorization procedure.

Lemma 3.7. Assume that $B$ satisfies the shrinking domain condition. Let $f$ be a non-identically vanishing function in $B$. Then for every set $E$, $\bar{V}_{2} \subset E \subset \mathbb{C} \backslash \bar{V}_{1}$, there exist $f_{1} \in B_{1}$ and $f_{2} \in B_{2}$ such that $f=f_{1} \cdot f_{2}$, furthermore $Z_{\Omega_{1}}\left(f_{1}\right)=Z_{\Omega}(f) \cap E$, and $Z_{\Omega_{2}}\left(f_{2}\right)=Z_{\Omega}(f) \backslash E$.

Proof: First of all we note that $Z_{\Omega}(f)$ is a countable subset of $\Omega$ without any limit point, since $f$ is not identically zero. In view of Weierstrass theorem (see [30, p. 303]), there exist $\varphi_{1} \in \mathcal{O}\left(\Omega_{1}\right)$ and $\varphi_{2} \in \mathcal{O}\left(\Omega_{2} \cup\{\infty\}\right.$ ) such that $Z_{\Omega_{1}}\left(\varphi_{1}\right)=Z_{\Omega}(f) \cap E$ and $Z_{\Omega_{2}}\left(\varphi_{2}\right)=Z_{\Omega}(f) \backslash E$, moreover, the zeros of $\varphi_{1} \cdot \varphi_{2}$ have the same multiplicities as those of $f$. Hence $f /\left(\varphi_{1} \cdot \varphi_{2}\right) \in(\mathcal{O}(\Omega))^{-1}$, the set of all invertible elements of $\mathcal{O}(\Omega)$. Let $U_{1}$ and $U_{2}$ be two finitely connected smoothly bordered domains satisfying the following inclusions, respectively,

$$
\bar{V}_{1} \subset U_{1} \subset \subset \Omega_{1}, \quad \bar{V}_{2} \subset U_{2} \subset \bar{U}_{2} \subset \Omega_{2},
$$

and such that $U_{1} \cup U_{2}=\mathbb{C}$ (note that $V_{1}$ and $V_{2}$ have already been fixed before Definition 3.5). Now, we see that $U=U_{1} \cap U_{2}$ is a finitely 
connected subdomain of $\Omega$ having $\partial U=\partial U_{1} \cup \partial U_{2}$ as its boundary. Recalling the projections $P_{1}$ and $P_{2}$ as introduced at the begining of section 3 , we notice that $P_{1}$ and $P_{2}$ extend continuously to projections $\mathcal{O}(U) \rightarrow \mathcal{O}\left(U_{1}\right)$ and $\mathcal{O}(U) \rightarrow \mathcal{O}_{0}\left(U_{2}\right)$, respectively, adding up to identity. Since $U$ is a finitely connected smoothly bordered domain, we can find rational functions $h_{1} \in\left(\mathcal{O}\left(U_{1}\right)\right)^{-1}$ and $h_{2} \in\left(\mathcal{O}\left(U_{2} \cup\right.\right.$ $\{\infty\}))^{-1}$ such that the winding number of $f /\left(\varphi_{1} \varphi_{2} h_{1} h_{2}\right)$ as an element of $(\mathcal{O}(U))^{-1}$ is zero around each component of $\mathbb{C} \backslash U$. This allows us to write $g=\log \left(f /\left(\varphi_{1} \varphi_{2} h_{1} h_{2}\right)\right) \in \mathcal{O}(U)$, therefore we have the following factorization of $f \in \mathcal{O}(U)$;

$$
\begin{aligned}
f=\varphi_{1} \varphi_{2} h_{1} h_{2} \exp (g) & =\varphi_{1} \varphi_{2} h_{1} h_{2} \exp \left(P_{1} g+P_{2} g\right) \\
& =\varphi_{1} h_{1} \exp \left(P_{1} g\right) \cdot \varphi_{2} h_{2} \exp \left(P_{2} g\right) \\
& =f_{1} \cdot f_{2}
\end{aligned}
$$

where $f_{1} \in \mathcal{O}\left(U_{1}\right)$ and $f_{2} \in \mathcal{O}\left(U_{2} \cup\{\infty\}\right)$. Since $f_{2}$ has no zeros in $\Omega_{1} \backslash U_{1}$, we have in fact $f_{1}=f / f_{2} \in \mathcal{O}\left(\Omega_{1}\right)$. To see that $f_{2}=f / f_{1} \in \mathcal{O}\left(\Omega_{2}\right)$, we note that $f_{1}$ has no zeros in $\Omega_{2} \backslash E$, and that $f$ and $f_{1}$ have the same zeros with the same multiplicities in $\Omega_{2} \cap E$. Moreover,

$Z_{\Omega_{1}}\left(f_{1}\right)=Z_{\Omega}\left(\varphi_{1}\right)=Z_{\Omega}(f) \cap E, \quad$ and $\quad Z_{\Omega_{2}}\left(f_{2}\right)=Z_{\Omega_{2}}\left(\varphi_{2}\right)=Z_{\Omega}(f) \backslash E$.

What therefore has to be shown is $f_{1} \in B_{1}$ and $f_{2} \in B_{2}$. By our choice of $E$, it is clear that $f_{1} \in\left(\mathcal{O}\left(\bar{V}_{1}\right)\right)^{-1}$, and $f_{2} \in\left(\mathcal{O}\left(\bar{V}_{2}\right)\right)^{-1}$, thus, in view of Proposition $1.5(\mathrm{~b})$, we obtain $f_{1} \in\left(M\left(B^{(2)}\right)\right)^{-1}$ and $f_{2} \in\left(M\left(B^{(1)}\right)\right)^{-1}$. Taking into account the fact that $f \in B \subset B^{(1)}$, we get

$$
f_{1}=\frac{1}{f_{2}} \cdot f \in B^{(1)} \cap \mathcal{O}\left(\Omega_{1}\right)=B_{1} .
$$

A similar argument reveals that $f_{2} \in B_{2}$.

The next lemma which has spectral theory flavour constitutes a half of ingredients of a proposition regarding the factorization of invariant subspaces of $B$ with index one which in turn will prove to be useful.

Lemma 3.8. Let $J$ be any multiplier invariant subspace of $B$ of index 1 and let $E$ be the set defined in the formulation of the previous lemma. Then there exist two multiplier invariant subspaces $J_{1}$ and $J_{2}$ of $B$ such that the following hold:

(a) $J=J_{1} \cap J_{2}$,

(b) $\sigma\left(M_{z}\left[B / J_{1}\right]\right)=\sigma\left(M_{z}[B / J]\right) \cap E$,

(c) $\sigma\left(M_{z}\left[B / J_{2}\right]\right)=\sigma\left(M_{z}[B / J]\right) \backslash E$. 
Proof: To simplify the notation, let us temporarily denote by $T$ the operator $M_{z}[B / J]$ defined earlier on $B / J$. We first assume that $J$ is an invariant subspace of $B$ with index one. What we really need is to decompose $T$ into summands with small spectra, which can be accomplished in the framework of classical holomorphic functional calculus. Recall that $\mathcal{O}(\sigma(T))$ denotes the algebra of all analytic functions on some neighbourhood of the compact set $\sigma(T)$ and let $U \supset \sigma(T)$ be an open (not neccessarily connected) set whose boundary $\partial U$ consists of a finite number of rectifiable Jordan curves oriented in the positive direction as customary in the theory of complex variables. Suppose $U \cup \partial U$ is contained in the domain of analyticity of a given $f \in \mathcal{O}(\sigma(T))$. Associate to $f$ the following operator on $B / J$ :

$$
f(T)=\frac{1}{2 \pi i} \int_{\partial U} f(\lambda)(\lambda-T)^{-1} d \lambda .
$$

Since $J$ has index one, it follows from $([\mathbf{2 7}$, Theorem 4.5$])$ that the spectrum of the operator $M_{z}[B / J]$ is discrete in $\Omega$. Consider now the isolated portions $\sigma_{1}=\sigma(T) \cap E$ and $\sigma_{2}=\sigma(T) \backslash E$ of the compact set $\sigma(T)$. Let $e_{1}$ be a function in $\mathcal{O}(\sigma(T))$ which is equal to 1 near $\sigma_{1}$ and equal to 0 near the other portion $\sigma_{2}$ and define $Q_{1}=e_{1}(T)$. Analogously one can consider the function $e_{2}$, which is equal to 1 near $\sigma_{2}$ and equal to 0 near $\sigma_{1}$, to obtain $Q_{2}=e_{2}(T)$. It is well-known that $Q_{1}$ and $Q_{2}$ are projections, simply because of $e_{1}^{2}=e_{1}$, and $e_{2}^{2}=e_{2}$. Moreover, since in a neighbourhood of $\sigma(T)$ we have $e_{1}+e_{2}=1$ and $e_{1} \cdot e_{2}=e_{2} \cdot e_{1}=0$, it follows that

$$
Q_{1}+Q_{2}=\text { identity, } \text { and } Q_{1} Q_{2}=Q_{2} Q_{1}=0 .
$$

These equalities imply that

$$
B / J=\operatorname{im} Q_{1} \oplus \operatorname{im} Q_{2}, \quad \operatorname{im} Q_{1}=\operatorname{ker} Q_{2}, \quad \text { and } \quad \operatorname{im} Q_{2}=\operatorname{ker} Q_{1},
$$

where $\operatorname{im} Q_{j}$ stands for the image of $B / J$ under $Q_{j}$ for $j=1,2$. For the direct sum, we note that if $x \in \operatorname{im} Q_{1} \cap \operatorname{im} Q_{2}$, then we have $Q_{1}(y)=$ $x=Q_{2}(z)$ for some $y$ and $z$ in $B / J$. Thus $y=Q_{1}^{2}(y)=Q_{1} Q_{2}(z)=0$, and hence $x=Q_{1}(y)=Q_{1}(0)=0$. Moreover, $\operatorname{im} Q_{1}$ and $\operatorname{im} Q_{2}$ are two closed invariant subspaces of $B / J$ with the property $\sigma\left(\left.T\right|_{\operatorname{im} Q_{j}}\right)=\sigma_{j}$, for $j=1,2[\mathbf{2 6}$, p. 84]. Put

$$
J_{2}=\left\{f \in B: f+J \in \operatorname{im} Q_{1}\right\} \supset J .
$$

It is obvious that $J_{2}$ is a closed invariant subspace of $B$, and that $\operatorname{im} Q_{1}=$ $J_{2} / J$. The closed invariant subspace $J_{1}$ of $B$ can be found in an analogous manner:

$$
J_{1}=\left\{f \in B: f+J \in \operatorname{im} Q_{2}\right\} .
$$


From the above the inclusion $J \subset J_{1} \cap J_{2}$ is obvious. For the reverse inclusion, suppose that $f$ is an element of the intersection of $J_{1}$ and $J_{2}$. Then $f+J \in \operatorname{im} Q_{1} \cap \operatorname{im} Q_{2}=\{0\}$, or equivalently $f \in J$. So far we have proved (a). To prove the other two statements of the lemma we first notice that $B / J_{1}$ is isomorphic to $\frac{B / J}{J_{1} / J}$, and secondly that because of $B / J=J_{1} / J \oplus J_{2} / J$ we also have $\frac{B / J}{J_{1} / J} \cong J_{2} / J$. Hence we have $B / J_{1} \cong J_{2} / J$. Now define $T_{1}: B / J_{1} \rightarrow B / J_{1}$ by $f+J_{1} \mapsto z f+J_{1}$, and $T_{2}: J_{2} / J \rightarrow J_{2} / J$ sending $f+J$ to $z f+J$. Since $T_{2}=\left.T\right|_{J_{2} / J}$ and $J_{2} / J=\operatorname{im} Q_{1}$, we get $\sigma\left(T_{2}\right)=\sigma_{1}$. But, we also have $\sigma\left(T_{1}\right)=\sigma\left(T_{2}\right)$, because of the isomorphism

$$
\Psi: J_{2} / J \rightarrow B / J_{1}
$$

which takes $f+J$ to $f+J_{1}$, for $f \in B$. Hence

$$
\begin{aligned}
\Psi\left(T_{2}(f+J)\right)=\Psi(z f+J)=z f+J_{1} & =T_{1}\left(f+J_{1}\right) \\
& =T_{1}(\Psi(f+J)) .
\end{aligned}
$$

Therefore $\Psi T_{2}=T_{1} \Psi$ or equivalently $T_{2}=\Psi^{-1} T_{1} \Psi$. Similarly, we obtain $\lambda-T_{2}=\Psi^{-1}\left(\lambda-T_{1}\right) \Psi$, for every $\lambda \in \mathbb{C}$. This means that $\lambda-T_{1}$ is invertible if and only if $\lambda-T_{2}$ is invertible, therefore $\sigma\left(T_{1}\right)=\sigma\left(T_{2}\right)$ and finally

$$
\sigma\left(M_{z}\left[B / J_{1}\right]\right)=\sigma\left(M_{z}\left[J_{2} / J\right]\right)=\sigma_{1},
$$

which proves part (b). Similarly one can prove part (c). So far, we have proved the lemma with the words "multiplier invariant" replaced by "invariant". Now let $J$ be a multiplier invariant subspace of $B$ of index one. We want to show that $J_{1}$ and $J_{2}$ are also multiplier invariant subspaces. Suppose that $f \in J_{1}$, then $f+J \in \operatorname{im} Q_{2}=\operatorname{ker} Q_{1}$. Assume that $\varphi \in M(B)$, and that $\gamma_{2}$ is a curve around $\sigma_{2}$ oriented in the positive direction. We can regard the operator $M_{\varphi} \in \mathcal{L}(B / J)$ both as left multiplication by $\varphi$, and as right multiplication by $\varphi$. Since the operator $M_{\varphi}=M_{\varphi}[B / J]$ commutes with $(\lambda-T)^{-1}$, it follows that

$$
\begin{aligned}
Q_{1} M_{\varphi} & =\left(\frac{1}{2 \pi i} \int_{\gamma_{2}}(\lambda-T)^{-1} d \lambda\right) M_{\varphi} \\
& =M_{\varphi}\left(\frac{1}{2 \pi i} \int_{\gamma_{2}}(\lambda-T)^{-1} d \lambda\right) \\
& =M_{\varphi} Q_{1} .
\end{aligned}
$$

Hence

$Q_{1}(\varphi f+J)=Q_{1} M_{\varphi}[B / J](f+J)=M_{\varphi}[B / J] Q_{1}(f+J)=M_{\varphi}[B / J](0)=0$, since $Q_{1}(f+J)=0$. This shows that $\varphi f \in J_{1}$, that is, $J_{1}$ is a multiplier invariant subspace. Similar reasoning shows that $J_{2}$ is also multiplier invariant. 
Lemma 3.9. Let $B$ be a Banach space of analytic functions on $\Omega$ satisfying the axioms (0-1)-(0-4), and let $J$ be an invariant subspace of B. If $g \in \mathcal{O}(\bar{\Omega})$, then

$$
g\left(M_{z}[B / J]\right)=M_{g}[B / J]
$$

where the right hand side is the operator of multiplication by $g$ on the quotient space $B / J$.

Proof: We first note that by Proposition 1.5(b), $g \in M(B)$ and hence the operator $M_{g}[B / J]$ is well-defined. Now, let $U$ be a finitely connected smoothly bordered domain containing $\bar{\Omega}$ and such that $\bar{U}$ lies in the domain of analyticity of $g$. Assuming that $\partial U$ is oriented in the positive direction, and that $h \in B$, we have

$$
\begin{aligned}
g\left(M_{z}[B / J]\right)(h(z)+J) & =\frac{1}{2 \pi i} \int_{\partial U} g(\lambda)\left(\lambda-M_{z}[B / J]\right)^{-1}(h(z)+J) d \lambda \\
& =\frac{1}{2 \pi i} \int_{\partial U}\left(\frac{g(\lambda) h(z)}{\lambda-z}+J\right) d \lambda \\
& =h(z)\left(\frac{1}{2 \pi i} \int_{\partial U} \frac{g(\lambda)}{\lambda-z} d \lambda+J\right) \\
& =h(z)(g(z)+J) \\
& =M_{g}[B / J](h(z)+J) .
\end{aligned}
$$

Note that for $\lambda \in \partial U$, we have $h(z) /(\lambda-z) \in B$, by the axiom (0-3).

Proposition 3.10. Assume that $B$ satisfies the shrinking domain condition. Let $J$ be a multiplier invariant subspace of $B$ having index one, and let $J_{1}$ and $J_{2}$ be as in Lemma 3.8. If $\mathcal{O}\left(\bar{\Omega}_{1}\right)$ and $\mathcal{O}\left(\bar{\Omega}_{2}\right)$ are dense in $B_{1}$ and $B_{2}$, respectively, then each $f \in J$ has a factorization $f=f_{1} \cdot f_{2}$, where $f_{1} \in J_{1} \cap B_{1}$ and $f_{2} \in J_{2} \cap B_{2}$.

Proof: Choose the functions $f_{1}$ and $f_{2}$ as in Lemma 3.7. What remains to be proved is $f_{1} \in J_{1}$ and $f_{2} \in J_{2}$. Since the proofs are similar, we shall just verify that $f_{1} \in J_{1}$. By assumption, for $f_{2} \in B_{2}$ we can find a sequence of functions $g_{k} \in \mathcal{O}\left(\bar{\Omega}_{2}\right)$ converging to $f_{2}$ in $B_{2}$. We recall that $B$ satisfies the shrinking domain condition, so we have $B_{2}=B^{(2)} \cap \mathcal{O}\left(\Omega_{2}\right)$. Consequently, $g_{k}$ tends to $f_{2}$ in $\mathcal{O}\left(\Omega_{2}\right) \subset \mathcal{O}\left(\sigma_{1}\right)$, where $\sigma_{1}$ is the spectrum of $M_{z}\left[B / J_{1}\right]$ as in Lemma 3.8. We know that if the space $\mathcal{O}\left(\Omega_{2}\right)$ is 
equipped with the uniform convergence topology, then the holomorphic functional calculus is continuous [31, p. 244]. Therefore

$$
g_{k}\left(M_{z}\left[B / J_{1}\right]\right) \rightarrow f_{2}\left(M_{z}\left[B / J_{1}\right]\right), \quad \text { as } \quad k \rightarrow \infty .
$$

Considering the fact proved in Lemma 3.9 that $g_{k}\left(M_{z}\left[B / J_{1}\right]\right)=$ $M_{g_{k}}\left[B / J_{1}\right]$, we get

$$
M_{g_{k}}\left[B / J_{1}\right]\left(f_{1}+J_{1}\right) \rightarrow f_{2}\left(M_{z}\left[B / J_{1}\right]\right)\left(f_{1}+J_{1}\right), \quad \text { as } \quad k \rightarrow \infty .
$$

We claim that $g_{k} f_{1} \rightarrow f$ in the norm of $B$. Putting off the proof of this claim to the end, we get

$$
f_{2}\left(M_{z}\left[B / J_{1}\right]\right)\left(f_{1}+J_{1}\right)=\left(\lim _{k \rightarrow \infty} g_{k} f_{1}\right)+J_{1}=f+J_{1}=0+J_{1},
$$

because $f \in J \subset J_{1}$. From the construction of $f_{2}$ we know that $Z_{\Omega_{2}}\left(f_{2}\right)=Z_{\Omega}(f) \backslash E$, (see Lemma 3.7); thus $f_{2}$ has no zeros in a neighbourhood of $\sigma_{1}$. This means that the operator $f_{2}\left(M_{z}\left[B / J_{1}\right]\right)$ is invertible and furthermore, its inverse in $\mathcal{L}\left(B / J_{1}\right)$ is $\left(1 / f_{2}\right)\left(M_{z}\left[B / J_{1}\right]\right)$ [31, p. 244]. Therefore from the equality (3-7) we have

$$
f_{1}+J_{1}=\left(1 / f_{2}\right)\left(M_{z}\left[B / J_{1}\right]\right)\left(0+J_{1}\right)=0+J_{1},
$$

or equivalently $f_{1} \in J_{1}$. We now turn to prove our postponed claim. We want to show that $\left\|g_{k} f_{1}-f\right\|_{B}=\left\|f_{1}\left(g_{k}-f_{2}\right)\right\|_{B} \rightarrow 0$, as $k \rightarrow \infty$. To see this, we first notice that

$$
\|f\|_{B} \asymp\|f\|_{B^{(1)}}+\|f\|_{B^{(2)}},
$$

where $B^{(1)}$ and $B^{(2)}$ are as in Definition 3.5. Here, the notation $\asymp$ is used to indicate that the two norms are equivalent.This estimate comes from the direct sums involved in $B, B^{(1)}$ and $B^{(2)}$ (see Definition 3.5) together with the closed graph theorem. It is also immediate from the definition of norm in the space of multipliers that

$$
\|f\|_{B^{(1)}} \leq\left\|f_{2}\right\|_{M\left(B^{(1)}\right)} \cdot\left\|f_{1}\right\|_{B^{(1)}},
$$

and

$$
\|f\|_{B^{(2)}} \leq\left\|f_{1}\right\|_{M\left(B^{(2)}\right)} \cdot\left\|f_{2}\right\|_{B(2)} .
$$


Since

$$
\left\|f_{1}\left(g_{k}-f_{2}\right)\right\|_{B} \asymp\left\|f_{1}\left(g_{k}-f_{2}\right)\right\|_{B^{(1)}}+\left\|f_{1}\left(g_{k}-f_{2}\right)\right\|_{B^{(2)}},
$$

we obtain

$$
\left\|f_{1}\left(g_{k}-f_{2}\right)\right\|_{B} \leq C\left(\left\|f_{1}\right\|_{B^{(1)}} \cdot\left\|g_{k}-f_{2}\right\|_{M\left(B^{(1)}\right)}+\left\|g_{k}-f_{2}\right\|_{B^{(2)}} \cdot\left\|f_{1}\right\|_{M\left(B^{(2)}\right)}\right),
$$

where $C$ is some constant. We know that $g_{k} \rightarrow f_{2}$ in the space $\mathcal{O}\left(\Omega_{2}\right)$, so $\left\|g_{k}-f_{2}\right\|_{M\left(B^{(1)}\right)} \rightarrow 0$, since $\mathcal{O}\left(\Omega_{2}\right) \subset M\left(B^{(1)}\right)$ by the holomorphic functional calculus. Also, $g_{k} \rightarrow f_{2}$ in the norm of $B_{2}$, and so that we get $\left\|g_{k}-f_{2}\right\|_{B^{(2)}} \rightarrow 0$. As a result, $\left\|f_{1}\left(g_{k}-f_{2}\right)\right\|_{B} \rightarrow 0$ as $k \rightarrow \infty$, which completes the proof of the proposition.

For an $M\left(B_{1}\right)$-invariant subspace $I$, we write

$$
\Lambda(I)=\operatorname{clos}(I \cdot M(B))
$$

for the smallest multiplier invariant subspace of $B$ containing $I$.

Theorem 3.11. Assume that $B=B_{1} \oplus B_{2}^{0}$ is a Banach space of analytic functions on $\Omega$ which contains 1 , satisfies the axioms (0-1)-(0-4), and the shrinking domain condition. Assume further that $\mathcal{O}\left(\bar{\Omega}_{1}\right)$ and $\mathcal{O}\left(\bar{\Omega}_{2}\right)$ are dense in $B_{1}$ and $B_{2}$, respectively. Then

(a) The mapping $I \mapsto \Lambda(I)$ is a bijection from the set of all $M\left(B_{1}\right)$ invariant subspaces $I$ with $\sigma\left(M_{z}\left[B_{1} / I\right]\right) \subset \Omega_{2}$ onto the set of all $M(B)$-invariant subspaces $J$ with $\sigma\left(M_{z}[B / J]\right) \subset \Omega_{2}$. Also

$$
\sigma\left(M_{z}[B / \Lambda(I)]\right)=\sigma\left(M_{z}\left[B_{1} / I\right]\right)
$$

and the inverse mapping is given by $J \mapsto J \cap B_{1}$.

(b) The mapping $L_{I}$ is a surjective module-homomorphism $B \rightarrow B_{1} / I$ which is canonical on $B_{1}$ and has kernel $\Lambda(I)$, for all $M\left(B_{1}\right)$ invariant subspaces $I$ with

$$
\sigma\left(M_{z}\left[B_{1} / I\right]\right) \subset \Omega_{2}
$$

(c) The quotient Banach spaces $B_{1} / I$ and $B / \Lambda(I)$ are canonically module-isomorphic for all $M\left(B_{1}\right)$-invariant subspaces $I$ with

$$
\sigma\left(M_{z}\left[B_{1} / I\right]\right) \subset \Omega_{2} .
$$


Proof: We start by proving (b); that $L_{I}$ is a surjective modulehomomorphism which is also canonical on $B_{1}$ follows from Proposition 3.3. What therefore has to be proved is that $\operatorname{ker} L_{I}=\Lambda(I)$. By Proposition 3.4(a), we have already proved that $\Lambda(I) \subset \operatorname{ker} L_{I}$. Writing ker $L_{I}=J$, we have from Proposition 3.4(b)

$$
J \cap B_{1}=\left(\operatorname{ker} L_{I}\right) \cap B_{1}=I .
$$

This equality makes the mapping $I \mapsto \operatorname{ker} L_{I}$ injective. Now, we want to show that $J \subset \Lambda(I)$. We intend to make use of our assumption that $\mathcal{O}\left(\bar{\Omega}_{2}\right)$ is dense in $B_{2}$. By Proposition 3.4(c), we know that

$$
\sigma\left(M_{z}[B / J]\right)=\sigma\left(M_{z}\left[B_{1} / I\right]\right) \subset \Omega_{2},
$$

thus Corollary 2.4 implies that the invariant subspace $J$ has index one. This allows us to apply Proposition 3.10 to $J$. According to Proposition 3.10, we know that each element $f$ of the multiplier invariant subspace $J$ has a product decomposition

$$
f=f_{1} \cdot f_{2}, \quad f_{1} \in J_{1} \cap B_{1}, \quad f_{2} \in J_{2} \cap B_{2},
$$

where $J_{1}, J_{2}$ are multiplier invariant subspaces of $B, J_{1} \cap J_{2}=J$, $\sigma_{J_{1}} \cap \sigma_{J_{2}}=\emptyset, \sigma_{J_{1}} \cup \sigma_{J_{2}}=\sigma_{J}$, and $\sigma_{J_{1}} \subset \Omega_{2}, \sigma_{J_{2}} \subset \Omega_{1}$. We may assume that $J_{1}=J$ and $J_{2}=B_{2}$, because we have made the assumption that the spectrum of the operator $M_{z}[B / J]$ lies in $\Omega \cup \partial \Omega_{1}$ so that the portion $\sigma_{2}$ of this spectrum in the situation of Proposition 3.10 consists of finitely many points, hence Proposition 2.5 implies that $J_{2}=p B$ for some polynomial $p$ with zeros in $\sigma_{J_{2}}$. Write $f_{2}=p g$ for some $g \in B$, and note that $p$ devides any element of $J_{2}$ (see Proposition 2.5), so that in fact we have $g \in B_{2}$. Therefore $f=\left(f_{1} \cdot p\right) \cdot g$, where $f_{1} \cdot p \in J_{1} \cap J_{2}=J$, and $g \in B_{2}$. But $f_{1} \cdot p \in B_{1}$ so that $f_{1} \cdot p \in J \cap B_{1}=I$, by Proposition 3.4(b), proving that

$$
J=\left(J \cap B_{1}\right) \cdot B_{2}=I \cdot B_{2} .
$$

We now turn to prove that $J \subset \Lambda(I)$. For $f \in J$, we have $f=f_{1} \cdot f_{2}$ where $f_{1} \in J \cap B_{1}=I$, and $f_{2} \in B_{2}$. Since, by assumption, $\mathcal{O}\left(\bar{\Omega}_{2}\right)$ is dense in $B_{2}$, it follows that $f_{2}$ can be approximated in $B_{2}$ by a sequence of elements of this space, say $f_{2, j}$. To show that $J \subset \Lambda(I)$, it suffices to verify that

$$
\left\|f-f_{1} f_{2, j}\right\|_{B}=\left\|f_{1}\left(f_{2}-f_{2, j}\right)\right\|_{B} \rightarrow 0, \quad \text { as } \quad j \rightarrow \infty
$$

because

$$
f_{1} f_{2, j} \in\left(J \cap B_{1}\right) \cdot M\left(B_{2}\right)=I \cdot M\left(B_{2}\right) \subset I \cdot M(B) .
$$


As in the proof of Proposition 3.10, we have

$$
\left\|f_{1}\left(f_{2}-f_{2, j}\right)\right\|_{B} \asymp\left\|f_{1}\left(f_{2}-f_{2, j}\right)\right\|_{B^{(1)}}+\left\|f_{1}\left(f_{2}-f_{2, j}\right)\right\|_{B^{(2)}},
$$

and therefore

$$
\begin{aligned}
& \left\|f_{1}\left(f_{2}-f_{2, j}\right)\right\|_{B} \\
& \quad \leq C\left(\left\|f_{1}\right\|_{B^{(1)}} \cdot\left\|f_{2}-f_{2, j}\right\|_{M\left(B^{(1)}\right)}+\left\|f_{2}-f_{2, j}\right\|_{B^{(2)}} \cdot\left\|f_{1}\right\|_{M\left(B^{(2)}\right)}\right),
\end{aligned}
$$

where $C$ is some constant. Since $f_{2, j} \rightarrow f_{2}$ in the space $\mathcal{O}\left(\Omega_{2}\right)$, we obtain $\left\|f_{2}-f_{2, j}\right\|_{M\left(B^{(1)}\right)} \rightarrow 0$, because $\mathcal{O}\left(\Omega_{2}\right) \subset M\left(B^{(1)}\right)$ by the holomorphic functional calculus, and finally $\left\|f_{2}-f_{2, j}\right\|_{B^{(2)}} \rightarrow 0$ by our assumption. Therefore we obtain $\left\|f_{1}\left(f_{2}-f_{2, j}\right)\right\|_{B} \rightarrow 0$, as $j \rightarrow \infty$ completing the proof of part (b).

To prove part (c), we note that for an $M\left(B_{1}\right)$-invariant subspace $I$ with condition $\sigma\left(M_{z}\left[B_{1} / I\right]\right) \subset \Omega_{2}$, the surjective module-homomorphism $L_{I}: B \rightarrow B_{1} / I$ induces an isomorphism

$$
\overline{L_{I}}: B / \operatorname{ker} L_{I}=B / \Lambda(I) \longrightarrow B_{1} / I .
$$

Since $L_{I}(f)=f+I$ for $f \in B_{1}$, it follows that $\left(\overline{L_{I}}\right)^{-1}: B_{1} / I \rightarrow B / \Lambda(I)$ has to coincide with the canonical homomorphism $B_{1} / I \rightarrow B / \Lambda(I)$. Note that for $f \in B_{1}$ we have $\overline{L_{I}}\left(f+\operatorname{ker} L_{I}\right)=L_{I}(f)$ if and only if $\left(\bar{L}_{I}\right)^{-1}\left(L_{I}(f)\right)=f+\operatorname{ker} L_{I}$, or

$$
\left(\overline{L_{I}}\right)^{-1}(f+I)=f+\operatorname{ker} L_{I}=f+\Lambda(I), \quad f \in B_{1} .
$$

Concerning the isomorphism $\overline{L_{I}}$, it should be noted that for every $f \in B$ and every $\varphi \in M(B)$ we have

$$
\begin{aligned}
\overline{L_{I}}(\varphi f+J)=L_{I}(\varphi f) & =L_{I, M}(\varphi) L_{I}(f) \\
& =L_{I, M}(\varphi) \overline{L_{I}}(f+J) .
\end{aligned}
$$

This together with the fact that $M(B)$ acts on $B / J$ according to

$$
\varphi \cdot(f+J)=\varphi f+J, \quad \varphi \in M(B) \text { and } f \in B,
$$

suggest that we might call $\overline{L_{I}}$ a multiplier module-isomorphism, that is, a surjective and injective multiplier module-homomorphism.

Regarding part (a), note that we have already shown, in part (b) above, that for any $M\left(B_{1}\right)$-invariant subspace $I$ with $\sigma\left(M_{z}\left[B_{1} / I\right]\right) \subset \Omega_{2}$ we have

$$
\operatorname{ker} L_{I}=\Lambda(I) \text {. }
$$


This together with Proposition 3.4(b) gives

$$
\Lambda(I) \cap B_{1}=\left(\operatorname{ker} L_{I}\right) \cap B_{1}=I,
$$

which in turn proves that the mapping $I \mapsto \Lambda(I)$ is injective. To prove the surjectivity of this mapping, let $J$ be an arbitrary $M(B)$-invariant subspace with $\sigma\left(M_{z}[B / J]\right) \subset \Omega_{2}$. As above, we may again assume that $J=J_{1}$ and $J_{2}=B_{2}$ where $J_{1}$ and $J_{2}$ are as in Proposition 3.10. Therefore we have $J=\left(J \cap B_{1}\right) \cdot B_{2}$. Let $f \in J$ be written as $f=f_{1} \cdot f_{2}$ where $f_{1} \in J \cap B_{1}$ and $f_{2} \in B_{2}$. By our assumption, we can approximate $f_{2} \in B_{2}$ by a sequence of functions in $\mathcal{O}\left(\bar{\Omega}_{2}\right)$. As in part (b), it turns out that

$$
f \in \operatorname{clos}\left\{\left(J \cap B_{1}\right) \cdot M\left(B_{2}\right)\right\}
$$

and hence

$$
J \subset \operatorname{clos}\left\{\left(J \cap B_{1}\right) \cdot M\left(B_{2}\right)\right\} \subset \operatorname{clos}\left\{\left(J \cap B_{1}\right) \cdot M(B)\right\} .
$$

This together with the obvious fact that $J \supset \operatorname{clos}\left\{\left(J \cap B_{1}\right) \cdot M(B)\right\}$ prove that we have in fact the equality

$$
J=\operatorname{clos}\left\{\left(J \cap B_{1}\right) \cdot M(B)\right\}=\Lambda\left(J \cap B_{1}\right),
$$

which in turn proves the surjectivity of $\Lambda$. Finally, we recall that the equality of the two spectra was shown in Proposition 3.4(c). Now, the proof of our theorem is complete.

Remark 3.12. Theorem 3.11 supplies a one-to-one correspondence between the multiplier invariant subspaces $J$ of $B$ with $\sigma\left(M_{z}[B / J]\right) \subset \Omega_{2}$ and the multiplier invariant subspaces $I$ of $B_{1}$ with $\sigma\left(M_{z}\left[B_{1} / I\right]\right) \subset \Omega_{2}$. What can we do when we wish to describe the structure of all multiplier invariant subspaces of index one in $B$ in terms of corresponding lattices of multiplier invariant subspaces in $B_{1}$ and $B_{2}$, respectively? We note first that by a suitable Möbius transformation, we may interchange the roles of $B_{1}$ and $B_{2}$. This means that under appropriate assumptions on $B, B_{1}$, and $B_{2}$, Theorem 3.11 also provides a one-to-one correspondence between the multiplier invariant subspaces $J$ of $B$ with $\sigma\left(M_{z}[B / J]\right) \subset \Omega_{1}$ and the multiplier invariant subspaces $I$ of $B_{2}$ with $\sigma\left(M_{z}\left[B_{2} / I\right]\right) \subset \Omega_{1}$. A general multiplier invariant subspace $J$ of $B$ of index one splits into $J=J_{1} \cap J_{2}$, where $J_{1}$ and $J_{2}$ are two other multiplier invariant subspaces of $B$ such that the spectra associated to the operators $M_{z}\left[B / J_{1}\right]$ and $M_{z}\left[B / J_{2}\right]$ are disjoint parts of the spectrum $\sigma\left(M_{z}[B / J]\right)$ in accordance with Lemma 3.8. By Theorem 3.11 (applied to $J_{1}$ and $J_{2}$, respectively), $J_{1}$ corresponds to $I_{1}=J_{1} \cap B_{1}$ and $J_{2}$ corresponds to $I_{2}=J_{2} \cap B_{2}$. 
So, to describe $J$, we essentially just need to understand $I_{1}$ and $I_{2}$. Moreover, by Proposition 3.10, each element $f \in J$ has a factorization $f=f_{1} \cdot f_{2}$, where $f_{1} \in I_{1}$ and $f_{2} \in I_{2}$. It follows that $I_{1}$ takes care of the boundary behavior of the functions in $J$ near $\partial \Omega_{1}$, and that $I_{2}$ takes care of the boundary behavior of the functions in $J$ near $\partial \Omega_{2}$. It should be pointed out that all known examples of multiplier invariant subspaces of index one involve boundary behavior (if we omit the rather trivial ones generated by polynomials, corresponding to finitely many zeros).

Acknowledgement. We thank the referee for calling our attention to the papers $[\mathbf{1 0}]$ and $[\mathbf{2 3}]$.

\section{References}

1. A. AbKar and H. Hedenmalm, Invariant subspaces in quasiBanach spaces of analytic functions, St. Petersburg Math. J. (to appear).

2. A. Aleman, Hilbert spaces of analytic functions between the Hardy and the Dirichlet space, Proc. Amer. Math. Soc. 115 (1992), 97-104.

3. A. Aleman and S. Richter, Simply invariant subspaces of $H^{2}$ of some multiply connected regions, Integral Equations Operator Theory 24 (1996), 127-155.

4. A. Aleman, S. Richter and W. T. Ross, Pseudocontinuations and the backward shift, Indiana Univ. Math. J. 47 (1998), 223-276.

5. A. Aleman, S. Richter and C. Sundberg, Beurling's theorem for the Bergman space, Acta Math. 177 (1996), 275-310.

6. C. Apostol, H. Bercovici, C. Foiaş and C. Pearcy, Invariant subspaces, dilation theory, and the structure of the predual of a dual algebra, J. Funct. Anal. 63 (1985), 369-404.

7. H. BerCovicI, The algebra of multiplication operators on Bergman spaces, Arch. Math. 48 (1987), 165-174.

8. A. Beurling, On two problems concerning linear transformations in Hilbert space, Acta Math. 81 (1949), 239-255.

9. L. Brown and A. L. Shields, Cyclic vectors in the Dirichlet space, Trans. Amer. Math. Soc. 285 (1984), 269-304.

10. K. C. Chan And A. L. Shields, Zero sets, interpolating sequences, and cyclic vectors for Dirichlet spaces, Michigan Math. J. 19 (1992), 289-307.

11. N. Dunford And J. T. Schwartz, "Linear Operators," Interscience Publishers, 1958. 
12. P. L. Duren, "Theory of $H^{p}$ Spaces," Academic Press, New York, 1970.

13. T. W. Gamelin, "Uniform Algebras," Prentice-Hall, Englewood Cliffs, N.J., 1969.

14. J. B. Garnett, "Bounded Analytic Functions," Academic Press, New York, 1981.

15. M. Hasumi, Invariant subspace theorems for finite Riemann surfaces, Canad. J. Math. 18 (1966), 240-255.

16. H. Hedenmalm, Bounded analytic functions and closed ideals, $J$. Anal. Math. 48 (1987), 142-166.

17. H. Hedenmalm, A comparison between the closed modular ideals in $l^{1}(w)$ and $L^{1}(w)$, Math. Scand. 58 (1986), 275-300.

18. H. Hedenmalm, Comparison of ideal structure in algebras of analytic functions of several complex variables, Math. Scand. 63 (1988), 305-314.

19. H. Hedenmalm, Spectral properties of invariant subspaces in the Bergman space, J. Funct. Anal. 116 (1993), 441-448.

20. H. Hedenmalm, An invariant subspace of the Bergman space having the codimension two property, J. Reine Angew. Math. 443 (1993), 1-9.

21. H. Hedenmalm, S. Richter and K. Seip, Interpolating sequences and invariant subspaces of given index in the Bergman spaces, J. Reine Angew. Math. 477 (1996), 13-30.

22. H. Helson, "Lectures on Invariant Subspaces," Academic Press, 1964.

23. D. Hitт, Invariant subspaces of $H^{2}$ of an annulus, Pacific J. Math. 134 (1988), 101-120.

24. K. Hoffman, "Banach Spaces of Analytic Functions," Dover Publications, Inc., 1988.

25. G. Kӧтне, "Topological Vector Spaces I," Springer-Verlag, 1969.

26. N. K. Nikol'skiI, "Treatise on the Shift Operator," SpringerVerlag, 1986.

27. S. Richter, Invariant subspaces in Banach spaces of analytic functions, Trans. Amer. Math. Soc. 304 (1987), 585-616.

28. S. Richter And A. L. Shields, Bounded analytic functions in the Dirichlet space, Math. Z. 198 (1988), 151-159.

29. H. L. Royden, Invariant subspaces of $H^{p}$ for multiply connected regions, Pacific J. Math. 134 (1988), 151-172.

30. W. Rudin, "Real and Complex Analysis," 3rd edition, McGraw- 
Hill, 1986.

31. W. Rudin, "Functional Analysis," McGraw-Hill, New York, 1973.

32. D. Sarason, The $H^{p}$ spaces of an annulus, Mem. Amer. Math. Soc. 56 (1965), Providence, Rhode Island.

33. G. D. TAYlor, Multipliers on $D_{\alpha}$, Trans. Amer. Math. Soc. 123 (1966), 229-240.

34. M. VoICHICK, Ideals and invariant subspaces of analytic functions, Trans. Amer. Math. Soc. 111 (1964), 493-512.

35. M. Voichick, Invariant subspaces on Riemann surfaces, Canad. J. Math. 18 (1966), 399-403.

36. D. V. YAKUBOVICH, Invariant subspaces of the operator of multiplication by $z$ in the space $E^{p}$ in a multiply connected region, $J$. Soviet Math. 61 (1992), 2046-2056.

\author{
Department of Mathematics \\ Lund University \\ Box 118 \\ 22100 Lund \\ SWEDEN \\ e-mail: ali@maths.lth.se \\ e-mail: haakan@maths.lth.se
}

Primera versió rebuda el 17 de març de 1998, darrera versió rebuda el 28 de setembre de 1998 